\title{
Measurement of inequality of opportunity based on counterfactuals
}

\author{
DIRK VAN DE GAER \\ Ghent University, CORE and IAE-CSIC \\ and \\ XAVIER RAMOS UAB, EQUALITAS and IZA
}

\begin{abstract}
The theoretical literature on inequality of opportunity formulates basic properties that measures of inequality of opportunity should have. Standard methods for the measurement of inequality of opportunity determine the inequality in counterfactual outcome distributions that are constructed by statistical methods. We show that, when standard parametric procedures are used to construct the counterfactuals, the choice of inequality measurement method and the statistical specification interact to determine whether the resulting measure of inequality of opportunity satisfies the basic properties.
\end{abstract}

Keywords Counterfactuals · Inequality Measurement · Opportunities

\section{Introduction}

Theories of equality of opportunity, see, e.g., Dworkin (1981a and 1981b), Arneson (1989), Cohen (1989), Roemer (1993 and 1998) put individual responsibility in the forefront in the assessment of the distribution of outcomes. Individuals' outcomes such as their income level, educational attainment or health status, are determined by two kinds of factors. On the one hand, there

Dirk Van de gaer acknowledges financial support from the FWO-Flanders, research project 3G079112. Both authors acknowledge financial support of projects ECO2013-46516-C4-1$\mathrm{R}$ and ECO2016-76506-C4-4-R (Ministerio de Ciencia y Tecnología) and Xavier Ramos acknowledges financial support of project 2014SGR-1279 (Direcció General de Recerca).

Dirk Van de gaer

Department of Economics, Ghent University, Sint-Pietersplein 6, B-9000 Gent, Belgium

Tel.: +32-9-2643482

Fax: +32-9-2648995

E-mail: Dirk.Vandegaer@ugent.be

Xavi Ramos

Departament d Economia Aplicada, Universitat Autònoma de Barcelona, Edifici B - Campus UAB, 8193 Bellaterra, Spain

E-mail: Xavi.Ramos@uab.cat 
are circumstances, factors that are beyond individuals' responsibility. On the other hand, there are efforts, factors for which individuals are responsible. Inequalities that are due to circumstances are deemed ethically unacceptable while those arising from efforts are not considered offensive. Hence, the outcome inequalities associated with these two factors should be treated differently. ${ }^{1}$

Following Fleurbaey and Peragine (2013) closely, we formulate two desirable properties that a measure of inequality of opportunity should have. The idea that inequalities that are due to unequal circumstances are offensive is reflected in the compensation principle: a Pigou-Dalton transfer reducing inequalities between individuals that have the same efforts (such that the resulting inequalities are due to circumstances) should decrease inequality of opportunity. The idea that inequalities that are due to differences in efforts are not offensive is reflected in the utilitarian reward principle: a Pigou-Dalton transfer reducing inequalities between individuals that have the same circumstances (such that the resulting inequalities are due to efforts) should not affect inequality of opportunity.

As a result, disentangling inequalities due to circumstances from those due to differences in efforts is key for empirical contributions. Both parametric and non-parametric methods are used for this purpose and to construct counterfactual outcome distributions in which all inequalities are due to circumstances or to efforts. Inequality of opportunity is then measured as the inequality in a counterfactual where all inequalities are only due to circumstances (the direct method), or as the difference between the inequality in the actual outcome distribution and the inequality in a counterfactual where all differences in inequality are only due to efforts (the indirect method). It is unclear whether these approaches result in measures of inequality of opportunity that are consistent with the basic principles mentioned above. The key point of this paper is that the choice of measurement method (direct versus indirect) and statistical method (non-parametric versus parametric, and for the latter also the parametric specification) interact to determine whether the resulting measure of inequality of opportunity satisfies the basic properties. For instance, the standard method of measuring inequality of opportunity by the inequality in the counterfactual that contains each individual's predicted outcome conditional on his circumstances satisfies utilitarian reward if the predicted value is obtained through a linear least squares regression. If, however, it is obtained through a loglinear least squares regression (as is common practice when the outcome of interest is income), the standard method fails to satisfy utilitarian reward. On the one hand, this key point helps to explain why many theorists are critical about much of the empirical literature. On the other hand, it makes researchers aware that the choice of the empirical specification can

1 Economists developed social criteria and ways to measure inequality of opportunity based on the dichotomy between circumstances and efforts. Overviews of this literature are Ferreira and Peragine (2016), Roemer and Trannoy (2015) and Ramos and Van de gaer (2016). 
have important consequences for the theoretical properties of their measure of inequality of opportunity.

The functional form used in parametric empirical analyses usually depends on the nature of the dependent variable and on the specification that one is used to in the literature. For inequality in PISA scores, for instance, Ferreira and Gignoux (2014) use a simple linear specification. So does Milanovic (2015) for years of schooling. For inequality of opportunity for income, a loglinear specification is almost universally used, see, e.g., Bourguignon et al. (2007), Ferreira and Gignoux (2011), Hassine (2012), Marrero and Rodríguez (2012), Singh (2012), Niehues and Peichl (2014) and Milanovic (2015). When the outcome of interest is binary or categorical, nonlinear probability models are commonly used. Foguel and Veloso (2014) and Trannoy et al. (2010), for instance, use a logit model to study inequality of access to education, and self-assessed health status, respectively, while Rosa Dias (2009) employs an ordered probit to examine self-assessed health status. In this paper we do not deal with nonlinear probability models, because it is much harder to define equalizing transfers for ordinal outcomes. The non-parametric methodology, developed by Checchi and Peragine (2010) and applied to university access by Brunori et al. (2012) constructs counterfactuals as group averages.

The structure of the paper is as follows. Section 2 introduces the notation, the basic distinction between direct and indirect measures of inequality of opportunity, and the different counterfactuals used in the empirical literature. The basic principles, compensation and utilitarian reward, are formalized in Section 3, while the results are presented in Section 4. Section ??sec:4:1 argues that indirect measures of inequality of opportunity can never satisfy utilitarian reward. Section 4.2 discusses the case when the counterfactual is constructed on the basis of a linear least squares estimate. It shows that some measures always satisfy one of the principles. We also derive sufficiency conditions under which other measures satisfy the principles, and show that some measures never satisfy some principles. For counterfactuals constructed on the basis of a loglinear least squares estimate, Section 4.3 demonstrates that the measures never satisfy the principles. Section 4.4 shows that measures based on the non-parametric methodology always satisfy one of the two principles. The conclusion is contained in Section 5.

\section{Measuring inequality of opportunity using counterfactuals}

Let $N=\{1, \ldots, n\}$, be the set of individuals. In theoretical work, $N$ is the population; in empirical work $N$ is typically a sample of individuals drawn from a population. The purpose of our work is to see how empirical approaches perform when used in the theorists' set-up. Therefore, it is best to think about $N$ as the population. We assume that the outcome we observe for individual $i$ is such that Pigou-Dalton transfers of this outcome can be meaningfully defined. We call this outcome individual $i$ 's income, $y_{i} \in \mathbb{R}_{++}$. Following the literature on equality of opportunity, we want to compensate her for some of 
her characteristics, while we want to hold her accountable for the vector of circumstances $\mathbf{c}_{\mathbf{i}} \in \mathbb{R}^{d^{C}}$, the latter in the vector of efforts $\mathbf{e}_{\mathbf{i}} \in \mathbb{R}^{d^{E}}{ }^{2}$ Define

$$
\mathbf{Y}=\left[\begin{array}{c}
y_{1} \\
\vdots \\
y_{n}
\end{array}\right], \mathbf{C}=\left[\begin{array}{c}
\mathbf{c}_{\mathbf{1}}{ }^{\prime} \\
\vdots \\
\mathbf{c}_{\mathbf{n}^{\prime}}
\end{array}\right], \mathbf{E}=\left[\begin{array}{c}
\mathbf{e}_{\mathbf{1}}{ }^{\prime} \\
\vdots \\
\mathbf{e}_{\mathbf{n}^{\prime}}
\end{array}\right],
$$

where $\mathbf{c}_{\mathbf{i}}$ ' and $\mathbf{e}_{\mathbf{i}}$ ' are the transpose of $\mathbf{c}_{\mathbf{i}}$ and $\mathbf{e}_{\mathbf{i}}$, respectively. Throughout we will assume that $\mathbf{C}$ and $\mathbf{E}$ are given and that the researcher is fully informed about the values of $\mathbf{Y}, \mathbf{C}$ and $\mathbf{E}$. This is not an innocuous assumption. The consequences of unobserved circumstances and efforts is discussed elsewhere, see, e.g., Roemer and Trannoy (2015) or Ramos and Van de gaer (2016), and is not the focus of the present paper, where we analyze a set-up that is as close as possible to that used in theoretical work. A dataset $\mathbf{d}$ is a triplet $(\mathbf{Y}, \mathbf{C}, \mathbf{E})$. The set of all possible data sets is

$$
D=\left\{(\mathbf{Y}, \mathbf{C}, \mathbf{E}): \mathbf{Y} \in \mathbb{R}_{++}^{n}, \mathbf{C} \in \mathbb{R}^{n \times d^{C}}, \mathbf{E} \in \mathbb{R}^{n \times d^{E}}\right\} .
$$

A measure of inequality of opportunity is a function $M: \mathbf{d} \in D \rightarrow \mathbb{R}$ such that $M(\mathbf{d})>M\left(\mathbf{d}^{\prime}\right)$ means that inequality of opportunity in $\mathbf{d}$ is higher than in $\mathbf{d}^{\prime}$. Let the function $I: \mathbf{Z} \in \mathbb{R}_{++}^{n} \rightarrow \mathbb{R}_{+}$be a measure of inequality, i.e., a function satisfying two properties. First, it satisfies the Pigou-Dalton transfer principle, meaning that, for all $\mathbf{Y}, \tilde{\mathbf{Y}} \in \mathbb{R}_{++}^{n}$ that are such that there exists $\delta \in \mathbb{R}_{++}$and $i, j \in N: \widetilde{y}_{i}=y_{i}-\delta \geq \widetilde{y}_{j}=y_{j}+\delta$, and for all $k \notin\{i, j\}$ : $\widetilde{y}_{k}=y_{k}, I(\tilde{\mathbf{Y}})<I(\mathbf{Y})$. Second, it satisfies anonymity, meaning that, for all $\mathbf{Y}, \widetilde{\mathbf{Y}} \in \mathbb{R}_{++}^{n}$ that are such that the vector $\widetilde{\mathbf{Y}}$ can be obtained from the vector $\mathbf{Y}$ by permuting its elements, $I(\widetilde{\mathbf{Y}})=I(\mathbf{Y})$.

The two most popular ways to measure inequality of opportunity are the direct and the indirect approach. Direct measures determine the amount of inequality of opportunity as the inequality in a counterfactual income distribution $\mathbf{Y}^{c}$ in which all inequalities due to differences in effort have been eliminated, such that the remaining inequality is solely due to differences in circumstances:

$$
M^{D}(\mathbf{d})=I\left(\mathbf{Y}^{c}(\mathbf{d})\right)
$$

Indirect measures determine the amount of inequality of opportunity by comparing the inequality in the actual distribution of income, $\mathbf{Y}$, to the inequality in a counterfactual income distribution $\mathbf{Y}^{E O}$ where there is no inequality of opportunity. This results in the measure

$$
M^{I}(\mathbf{d})=I(\mathbf{Y})-I\left(\mathbf{Y}^{E O}(\mathbf{d})\right) .
$$

To compute either of these measures a counterfactual distribution of income has to be constructed using the information in the dataset $\mathbf{d}$. This can be

\footnotetext{
2 In most applications, some characteristics are categorical; they are taken on board by dummy variables. This does not affect any of the analysis that follows, except that taking average values of characteristics as reference values (see Section 4.2) becomes hard to defend. In such a case it makes more sense to take for instance the median as a reference.
} 
done either with a parametric or a non-parametric approach. Three types of specifications have been used to construct counterfactual distributions parametrically. Individual $i$ 's income, $y_{i}$, is assumed to depend on her circumstances $\mathbf{c}_{\mathbf{i}}$ and her efforts, $\mathbf{e}_{\mathbf{i}}$, such that

$$
y_{i}=g\left(\mathbf{c}_{\mathbf{i}}, \mathbf{e}_{\mathbf{i}}\right) \quad \text { where } \quad g: \mathbb{R}^{d^{C}} \times \mathbb{R}^{d^{E}} \rightarrow \mathbb{R}_{++} .
$$

As the functional form $g$ is unknown, the parametric approach imposes a functional form to estimate the equation, yielding the function

$$
\widehat{g}\left(\mathbf{c}_{\mathbf{i}}, \mathbf{e}_{\mathbf{i}}, u_{i}\right) \quad \text { where } \widehat{g}: \mathbb{R}^{d^{C}} \times \mathbb{R}^{d^{E}} \times \mathbb{R} \rightarrow \mathbb{R}_{++} .
$$

The effect of specification errors go into the estimated random term, $\widehat{u}_{i}$, which is defined implicitly by the equation $y_{i}=\widehat{g}\left(\mathbf{c}_{\mathbf{i}}, \mathbf{e}_{\mathbf{i}}, \widehat{u}_{i}\right)$. Its estimate is determined by the chosen functional form $\widehat{g}$ and the dataset $\mathbf{d}$. Some counterfactuals treat it as a circumstance, others as an effort (see below). Other counterfactuals are based on estimates of incomes as a function of, alternatively circumstances and random variation, or efforts and random variation:

$$
\begin{aligned}
& \widehat{g}^{C}\left(\mathbf{c}_{\mathbf{i}}, u_{i}^{C}\right) \quad \text { where } \widehat{g}^{C}: \mathbb{R}^{d^{C}} \times \mathbb{R} \rightarrow \mathbb{R}_{++}, \\
& \widehat{g}^{E}\left(\mathbf{e}_{\mathbf{i}}, u_{i}^{E}\right) \quad \text { where } \widehat{g}^{E}: \mathbb{R}^{d^{E}} \times \mathbb{R} \rightarrow \mathbb{R}_{++} .
\end{aligned}
$$

In the first (second) equation, the effect of omitted efforts (circumstances) are taken over by circumstances (efforts) to the extent that these two are correlated. The rest of their effect as well as specification errors go into the estimated random term, $\widehat{u}_{i}^{C}\left(\widehat{u}_{i}^{E}\right)$, which is defined implicitly by the equation $y_{i}=\widehat{g}^{C}\left(\mathbf{c}_{\mathbf{i}}, \widehat{u}_{i}^{C}\right)\left(y_{i}=\widehat{g}^{E}\left(\mathbf{e}_{\mathbf{i}}, \widehat{u}_{i}^{E}\right)\right)$.

The following parametric counterfactuals have been or can be proposed for the direct approach:

$$
\begin{gathered}
y_{i}^{c 1}=\widehat{g}^{C}\left(\mathbf{c}_{\mathbf{i}}, 0\right), \\
y_{i}^{c 2}=\widehat{g}^{E}\left(\overline{\mathbf{e}}, \widehat{u}_{i}^{E}\right), \\
y_{i}^{c 3}=\widehat{g}\left(\mathbf{c}_{\mathbf{i}}, \overline{\mathbf{e}}, 0\right), \\
y_{i}^{c 4}=\widehat{g}\left(\mathbf{c}_{\mathbf{i}}, \overline{\mathbf{e}}, \widehat{u}_{i}\right), \\
y_{i}^{c 5}=\frac{1}{\left|N_{i \cdot}\right|} \sum_{k \in N_{i} .} \widehat{g}\left(\mathbf{c}_{\mathbf{k}}, \mathbf{e}_{\mathbf{k}}, 0\right),
\end{gathered}
$$

where $N_{i} .=\left\{k \in N\right.$ such that $\left.\mathbf{c}_{\mathbf{k}}=\mathbf{c}_{\mathbf{i}}\right\}$, the set containing all individuals that have the same circumstances as individual $i ; \mid N_{i}$. $\mid$ is the cardinality of that set. In (7) - (9), $\overline{\mathbf{e}}$ is a vector of reference values for efforts. Use of counterfactual (6) implies that one measures the inequality that is due to circumstances, including the indirect correlation between circumstances and efforts. Counterfactual (7) measures all inequalities that are due to circumstances and random terms that are not correlated with effort. In (8), differences in random terms are treated as efforts (i.e. inequalities due to differences in random terms are legitimate), 
while in (9) they are treated as circumstances. ${ }^{3}$ Counterfactual (10) is similar in spirit to (8), but instead of taking a reference value for effort, the average outcome of each circumstance group is taken. The most popular counterfactual is $\mathbf{Y}^{c 1}$; it was used, e.g., by Rosa Dias (2009), Ferreira and Gignoux (2011), Ferreira and Gignoux (2014), Brunori et al. (2012), Marrero and Rodríguez (2012), Foguel and Veloso (2014) and Niehues and Peichl (2014). Fleurbaey and Schokkaert (2009) suggest to use $\mathbf{Y}^{c 3}$ and $\mathbf{Y}^{c 4}$. Pistolesi (2009) used $\mathbf{Y}^{c 4}$. We are unaware of applications of $\mathbf{Y}^{c 2}$ and $\mathbf{Y}^{c 5}$.

Non-parametric procedures rely on averaging. The non-parametric counterfactual for the direct approach becomes

$$
y_{i}^{c 6}=\frac{1}{\left|N_{i}\right|} \sum_{k \in N_{i} .} y_{k},
$$

which is the average income of all those having the same circumstances as individual $i$. It was proposed in Van de gaer (1993) and developed in Checchi and Peragine (2010).

Changing some of the elements used to construct the parametric counterfactuals would/could suggest other possible counterfactuals. One could in (6) replace the zero by $\widehat{u}_{i}^{C}$. However, $\widehat{g}^{C}\left(\mathbf{c}_{\mathbf{i}}, \widehat{u}_{i}^{C}\right)=y_{i}$ and counterfactual income would be equal to actual income. Similarly in (10) the zero could be replaced by $\widehat{u}_{k}$, but, as $\widehat{g}\left(\mathbf{c}_{\mathbf{k}}, \mathbf{e}_{\mathbf{k}}, \widehat{u}_{i}\right)=y_{k}$, the counterfactual reduces to the nonparametric counterfactual (11). Once could in (7) replace $\widehat{u}_{i}^{E}$ by a zero, but then everybody would have the same counterfactual income. In both (7) and (9) the choice of reference effort could be replaced by an averaging procedure as in (10). In both cases, the counterfactual reduces to the non-parametric counterfactual (11).

For the indirect measurement approach the following parametric counterfactuals have been or can be proposed:

$$
\begin{gathered}
y_{i}^{E O 1}=\widehat{g}^{E}\left(\mathbf{e}_{\mathbf{i}}, 0\right), \\
y_{i}^{E O 2}=\widehat{g}^{C}\left(\overline{\mathbf{c}}, \widehat{u}_{i}^{C}\right), \\
y_{i}^{E O 3}=\widehat{g}\left(\overline{\mathbf{c}}, \mathbf{e}_{\mathbf{i}}, 0\right), \\
y_{i}^{E O 4}=\widehat{g}\left(\overline{\mathbf{c}}, \mathbf{e}_{\mathbf{i}}, \widehat{u}_{i}\right), \\
y_{i}^{E O 5}=\frac{1}{\left|N_{\cdot i}\right|} \sum_{k \in N_{\cdot i}} \widehat{g}\left(\mathbf{c}_{\mathbf{k}}, \mathbf{e}_{\mathbf{k}}, 0\right),
\end{gathered}
$$

where $N_{. i}=\left\{k \in N\right.$ such that $\left.\mathbf{e}_{\mathbf{k}}=\mathbf{e}_{\mathbf{i}}\right\}$, the set of individuals having the same efforts as individual $i ;\left|N_{. i}\right|$ is the cardinality of that set. In (13) - (15) $\overline{\mathbf{c}}$ is a vector of reference values for circumstances. The inequality in counterfactual (12) measures the inequality that is due to the direct effect of efforts as well

\footnotetext{
3 As in actual applications it is unclear whether the $u_{i}$ should be treated as a circumstance or effort, Fleurbaey and Schokkaert (2009) suggest to compute inequality of opportunity in both cases. This holds, of course, also when using an indirect measure of inequality of opportunity.
} 
as the part that is due to the correlation between efforts and circumstances. The inequality in counterfactual (13) reflects all inequalities that are due to efforts and random terms that are not correlated with circumstances. In (14), differences in random terms are treated as circumstances, while in (15) they are treated as efforts. Counterfactual (16) is similar in spirit to (14), but instead of taking a reference value for circumstances, the average outcome of each effort group is taken. Counterfactual $\mathbf{Y}^{E O 1}$ was used in Trannoy et al. (2010), $\mathbf{Y}^{E O 2}$ was used in Bourguignon et al. (2007), Singh (2012) and Hassine (2012). Fleurbaey and Schokkaert (2009) suggested $\mathbf{Y}^{E O 3}$ and $\mathbf{Y}^{E O 4}$. Brunori et al. (2012) applied $\mathbf{Y}^{E O 3}$ and $\mathbf{Y}^{E O 4}$. The latter was also applied by Bourguignon et al. (2007) and Pistolesi (2009). We are unaware of applications of $\mathbf{Y}^{E O 5}$.

Also in the indirect approach a non-parametric counterfactual can be constructed. The non-parametric counterfactual for the indirect approach becomes

$$
y_{i}^{E O 6}=\frac{1}{\left|N_{\cdot i}\right|} \sum_{k \in N_{\cdot i}} y_{k}
$$

which is the average income of all those having the same effort as individual $i$. Checchi and Peragine (2010) proposed this counterfactual.

Also here changing some of the elements present in the construction of the parametric counterfactuals suggests other possible counterfactuals. One could in (12) replace the zero by $\widehat{u}_{i}^{E}$. However, $\widehat{g}^{E}\left(\mathbf{c}_{\mathbf{i}}, \widehat{u}_{i}^{E}\right)=y_{i}$ and counterfactual income would be equal to actual income. Similarly in (16) the zero could be replaced by $\widehat{u}_{k}$, but, as $\widehat{g}\left(\mathbf{c}_{\mathbf{k}}, \mathbf{e}_{\mathbf{k}}, \widehat{u}_{i}\right)=y_{k}$, the counterfactual reduces to the non-parametric counterfactual (17). Once could in (13) replace $\widehat{u}_{i}^{C}$ by a zero, but then everybody would have the same counterfactual income. In both (13) and (15) the choice of reference circumstance could be replaced by an averaging procedure as in (16). In both cases, the counterfactual reduces to the non-parametric counterfactual (17).

\section{Measurement perspective}

Both theorists and empiricists use the information in the dataset $\mathbf{d}$ to construct a measure of inequality of opportunity. The empiricists use the dataset $\mathbf{d}$ in order to obtain a good estimate of parametric relationship (3), (4) or (5) to construct the counterfactuals or estimate the counterfactuals non-parametrically. Next, they compute a direct or indirect measure of inequality of opportunity, respectively (1) or (2).

The theorists take a measurement theory perspective. It requires that the measure of inequality of opportunity responds to changes in the dataset in a way compatible to the intuitions prescribed by equality of opportunity principles. Let the set of all datasets compatible with $\mathbf{Y}, \mathbf{C}$ and $\mathbf{E}$ be

$$
\Delta=\left\{(\mathbf{X}, \mathbf{C}, \mathbf{E}): \mathbf{X} \in \mathbb{R}_{++}^{n} \text { and } \sum_{i=1}^{n} x_{i}=\sum_{i=1}^{n} y_{i}\right\} .
$$


The domain $\Delta \subset D$. It keeps not only the set of individuals fixed, but also their characteristics $\mathbf{C}$ and $\mathbf{E}$, as well as the total income. It allows us to state how the measure of inequality of opportunity should respond to income redistributions between individuals. As both the direct and indirect approach use standard inequality measures to measure the inequality in the counterfactual distribution, we are interested in normative equality of opprtunity properties involving Pigou-Dalton transfers of income. Two prominant properties involving Pigou-Dalton transfers have been proposed in the literature (see, e.g., Fleurbaey and Peragine, 2013).

The first property, compensation, requires that, when 2 individuals $i$ and $j$ have the same efforts, and $i$ has a higher level of income than $j$, then transferring an amount of money $\delta$ from $i$ to $j$, without resulting in $i$ having a lower income than $j$, decreases inequality of opportunity, as their income difference, which is entirely due to circumstances, diminishes. ${ }^{4}$

$\mathbf{C O M}$ (Compensation) For all $\mathbf{d}=(\mathbf{Y}, \mathbf{C}, \mathbf{E})$ and $\widetilde{\mathbf{d}}=(\widetilde{\mathbf{Y}}, \mathbf{C}, \mathbf{E}) \in \Delta$ that are such that there exist $\delta \in \mathbb{R}_{++}$and $i, j \in N: \mathbf{e}_{\mathbf{i}}=\mathbf{e}_{\mathbf{j}}$ and $\widetilde{y}_{i}=y_{i}-\delta \geq \widetilde{y}_{j}=$ $y_{j}+\delta$, and for all $k \notin\{i, j\}: \widetilde{y}_{k}=y_{k}$, we have $M(\widetilde{\mathbf{d}})<M(\mathbf{d})$.

The idea that people are responsible for their efforts can be expressed by a second property, utilitarian reward, which also makes a statement about the effect of a particular kind of Pigou-Dalton transfer on inequality of opportunity. The starting point is that one should be neutral towards income differences that are only due to differences in efforts. Hence, when individuals $i$ and $j$ have the same circumstances and $i$ has a higher income than $j$, then, as the difference in incomes is due to efforts only, transferring an amount of money $\delta$ from $i$ to $j$ without resulting in $i$ having a lower income than $j$, should not affect inequality of opportunity. ${ }^{5}$

UR (Utilitarian Reward) For all $\mathbf{d}=(\mathbf{Y}, \mathbf{C}, \mathbf{E})$ and $\widetilde{\mathbf{d}}=(\widetilde{\mathbf{Y}}, \mathbf{C}, \mathbf{E}) \in \Delta$ that are such that there exists $\delta \in \mathbb{R}_{++}$and $i, j \in N: \mathbf{c}_{\mathbf{i}}=\mathbf{c}_{\mathbf{j}}$ and $\widetilde{y}_{i}=y_{i}-\delta \geq$ $\widetilde{y}_{j}=y_{j}+\delta$, and for all $k \notin\{i, j\}: \widetilde{y}_{k}=y_{k}$, we have $M(\widetilde{\mathbf{d}})=M(\mathbf{d})$.

We know from the literature on fair compensation that it is very difficult to reconcile compensation and reward principles (see, e.g., Bossert, 1995, Fleurbaey, 1995, Fleurbaey, 2008 or Fleurbaey and Maniquet, 2011). The same is true here: $\mathrm{COM}$ and UR are incompatible. To see this, consider four individuals: $N=\{1,2,3,4\}, \mathbf{c}_{\mathbf{1}}=\mathbf{c}_{\mathbf{2}}, \mathbf{c}_{\mathbf{3}}=\mathbf{c}_{\mathbf{4}}, \mathbf{e}_{\mathbf{1}}=\mathbf{e}_{\mathbf{3}}, \mathbf{e}_{\mathbf{2}}=\mathbf{e}_{\mathbf{4}}$, incomes $y_{1}, y_{2}, y_{3}$ and $y_{4}$ such that $y_{1}<y_{4}<y_{3}<y_{2}$ and a transfer $\delta \leq\left(y_{3}-y_{4}\right) / 2$ such that

\footnotetext{
4 Fleurbaey and Peragine (2013) labelled this property "Ex-Post Compensation". In the Conclusion we discuss the alternative "Ex-Ante Compensation" principle.

5 This principle has also been called "Utilitarianism for Equal Circumstances" by Fleurbaey (2008). We discuss an alternative, "Liberal reward" in the Conclusion. It has to be observed that not everyone in the literature endorses the reward principles; several authors suggest that only compensation is the core part of opportunity egalitarianism. See Roemer and Trannoy (2015) for a discussion.
} 
it satisfies the conditions in the properties we are using. The following Table gives the income distribution after the transfer.

Table 1 Incompatibility between COM and UR

\begin{tabular}{ccc}
\hline & $\mathbf{e}_{\mathbf{1}}=\mathbf{e}_{\mathbf{3}}$ & $\mathbf{e}_{\mathbf{2}}=\mathbf{e}_{\mathbf{4}}$ \\
\hline $\mathbf{c}_{\mathbf{1}}=\mathbf{c}_{\mathbf{2}}$ & $y_{1}+\delta$ & $y_{2}-\delta$ \\
$\mathbf{c}_{\mathbf{3}}=\mathbf{c}_{\mathbf{4}}$ & $y_{3}-\delta$ & $y_{4}+\delta$ \\
\hline Note: $y_{1}<y_{4}<y_{3}<y_{2}$ and $\delta \leq\left(y_{3}-y_{4}\right) / 2$.
\end{tabular}

Considering the transfers column-wise the new income distribution arises after two Pigou-Dalton transfers between individuals having the same efforts. By COM, inequality of opportunity decreased. Considering the transfers rowwise the new income distribution arizes after two Pigou-Dalton transfers between individuals having the same circumstances. By UR inequality of opportunity did not change. As the two properties judge the change in the income distribution differently, they are incompatible.

Hence we know that there does not exist any measure of inequality of opportunity that satisfies both COM and UR. Confronted with such an incompatibility, the standard approach in the theoretical literature is to weaken (at least) one of the properties to obtain properties that are compatible. That, however, is not the focus of our paper. Instead, we turn to the question whether, or under which conditions, the procedures that are most frequently used in the empirical literature satisfy the basic properties COM or UR.

\section{Results}

\subsection{Impossibility}

For indirect measures of inequality of opportunity, UR requires that the change in measured inequality in the counterfactual induced by a Pigou-Dalton transfer between individuals with the same circumstances exactly equals the change in measured inequality in the actual distribution of income. This can only hold for all inequality measures if the counterfactual equals the actual income distribution, which goes against the idea that the counterfactual represents a situation with equality of opportunity, a situation in which all inequalities are due to differences in efforts only. This results in the following impossibility.

Proposition 1 No indirect measure of inequality of opportunity can satisfy UR.

\subsection{Linear least squares}

Define average income $\mu_{Y}=\frac{1}{n} \sum_{j=1}^{n} y_{j}$, the average value of circumstance $k, \mu_{C k}=\frac{1}{n} \sum_{j=1}^{n} c_{j k}$ and $\mu_{\mathbf{C}}$ the $d^{C}-$ dimensional vector containing $\mu_{C k}$ as 
$k$-th element for all $k \in\left\{1, \ldots, d^{C}\right\}$. The average value for effort $q, \mu_{E q}=$ $\frac{1}{n} \sum_{j=1}^{n} e_{j q}$, and $\mu_{\mathbf{E}}$ is the $d^{E}-$ dimensional vector containing the $\mu_{E q}$ as $q$ -th element for all $q \in\left\{1, \ldots, d^{E}\right\}$. Let $\mathbf{E}_{\mathbf{i}}$ be the $n$-dimensional vector with zeros everywhere, except for its $i-t h$ element which equals one, $\iota$ the $n$ - dimensional vector of ones, $\mathbf{X}^{C}=\mathbf{C}-\iota \mu_{\mathbf{C}}, \mathbf{X}^{E}=\mathbf{E}-\iota \mu_{\mathbf{E}}, \mathbf{X}^{C E}=$ $\left[\begin{array}{ll}\mathbf{X}^{C} & \mathbf{X}^{E}\end{array}\right], \mathbf{Y}^{D}=\mathbf{Y}-\iota \mu_{Y}$,

$$
\mathbf{U}^{A}=\left[\begin{array}{c}
u_{1}^{A} \\
\vdots \\
u_{n}^{A}
\end{array}\right] \text { and } \mathbf{V}^{A}=\left[\begin{array}{c}
v_{1}^{A} \\
\vdots \\
v_{n}^{A}
\end{array}\right]
$$

where $A \in\{C, E, C E\}$. The equations that have to be estimated to construct the counterfactuals are, in deviational form,

$$
\mathbf{Y}^{D}=\mathbf{X}^{A} \beta^{A}+\mathbf{U}^{A} .
$$

The corresponding least squares estimator of the coefficient vector $\beta^{A}$ is

$$
\mathbf{b}^{A}=\left(\mathbf{X}^{A \prime} \mathbf{X}^{A}\right)^{-1} \mathbf{X}^{A \prime} \mathbf{Y}^{D}
$$

Performing a Pigou-Dalton transfer $\delta$ from observation $i$ to $j$ changes the least squares estimates of the coefficient vector, which affects the constructed counterfactuals and, thereby, the values of direct and indirect measures of inequality of opportunity. The following Lemma gives the relationship between $\widetilde{\mathbf{b}}^{A}$, the least squares estimate after the Pigou-Dalton transfer, and $\mathbf{b}^{A}$.

Lemma 1 A Pigou-Dalton transfer $\delta$ from observation $i$ to $j$ changes the least squares estimator of the coefficient vector into $\widetilde{\mathbf{b}}^{A}$, given by

$$
\widetilde{\mathbf{b}}^{A}=\mathbf{b}^{A}+\frac{\delta}{n}\left(\mathbf{S}^{A}\right)^{-1}\left(\mathbf{x}_{\mathbf{j}}{ }^{A}-\mathbf{x}_{\mathbf{i}}{ }^{A}\right),
$$

with $\mathbf{S}^{A}=\frac{1}{n} \mathbf{X}^{A \prime} \mathbf{X}^{A}, \mathbf{x}_{\mathbf{j}}{ }^{A}=\mathbf{X}^{A \prime} \mathbf{E}_{\mathbf{j}}$ and $\mathbf{x}_{\mathbf{i}}{ }^{A}=\mathbf{X}^{A \prime} \mathbf{E}_{\mathbf{i}}$.

The Lemma shows which elements will be important to determine the effect of the Pigou-Dalton transfer. First, observe that $\mathbf{x}_{\mathbf{j}}{ }^{A}$ gives the $j-t h$ column of the matrix $\mathbf{X}^{A \prime}$, which contains the values for the variables of individual $j$ in the matrix $\mathbf{X}^{A \prime}$. Hence, if the transfer takes place between two individuals with exactly the same values for the variables in $\mathbf{X}^{A}$, the estimate of the coefficient vector will not change. Second, observe that $\mathbf{S}^{A}$ is the covariance matrix of the variables in $\mathbf{X}^{A}$. The inverse of this covariance matrix enters the expression in Lemma 1. If $\mathbf{X}^{A}$ contains only one variable, $\mathbf{S}^{A}$ is the variance of that variable, such that $\left(\mathbf{S}^{A}\right)^{-1}$ is a positive scalar. If both circumstances and efforts are included in the matrix $\mathbf{X}^{A}$, and these variables are not correlated, both $\mathbf{S}^{A}$ and its inverse are block-diagonal. This implies that the estimated coefficient vector of circumstances is not affected by differences in efforts between $i$ and $j$, and the estimated coefficient vector of efforts is not affected by differences in circumstances between $i$ and $j$. 
We want to establish whether, with counterfactuals determined by least squares estimate (19), the measures of inequality of opportunity defined in Section 3 respond to Pigou-Dalton transfers as required by properties COM and UR. We find that few measures obey one of the properties, others don't and still others satisfy properties under some assumptions. In particular the following assumptions turn out to be helpful.

C1M Circumstances are one-dimensional and the transfer goes from someone with better circumstances to someone with worse circumstances: $d^{C}=1$ and $c_{i 1}>c_{j 1}$.

$\mathbf{E} \boldsymbol{\mu}$ Reference efforts are equal to average efforts: $\overline{\mathbf{e}}=\mu_{\mathbf{E}}$.

$\mathbf{C} \boldsymbol{\mu}$ Reference circumstances are equal to average circumstances: $\overline{\mathbf{c}}=\mu_{\mathbf{C}}$.

COR0 Circumstances and efforts are not correlated.

One-dimensionality of circumstances is a very strong assumption to make. However, observe that in the literature on intergenerational mobility, one often draws lessons about inequality of opportunity (see O'Neill et al., 2000, and, for a recent example Chetty et al., 2014), which requires a one-dimensional view of circumstances. In addition, C1M requires monotonicity: the transfer goes from an individual with better circumstances and higher income to someone with worse circumstances and lower income. The determination of the value for reference efforts or circumstances is a tedious issue in the literature. ${ }^{6}$ In much of the empirical literature, reference values are simply set to their sample means, without much justification, see, e.g., Bourguignon et al. (2007), Pistolesi (2009), Ferreira and Gignoux (2011) or Singh (2012). There has been some debate about the implications of the correlation between efforts and circumstances. Roemer (1993 and 1998) argued that normatively relevant effort has to be measured such that it is, by construction, not correlated with circumstances since it is very hard to hold people responsible for efforts if they are correlated with circumstances, which are, by definition, not under individual control. Others, e.g., Rawls (1971), Dworkin (1981a and 1981b), Van Parijs (1995) and Fleurbaey (2008) have argued that people should be responsible for their tastes, even if these are correlated with their circumstances.

The following Proposition, proven in Appendix A.2, lists the properties of the measures of inequality of opportunity.

Proposition 2 Using a linear specification and the least squares estimate of the coefficient vector to construct the counterfactual, the following Table gives sufficient conditions for the measures of inequality of opportunity to satisfy COM or UR.

6 There are not many theoretical results about the consequences of the choice of reference values -for an exception see Luttens and Van de gaer (2007). To solve the arbitrariness of the choice of reference value Ramos and Van de gaer (2016) propose an averaging procedure. García-Gómez et al. (2013) propose to minimize the extent to which the theoretical principles are violated. 
Table 2 Conditions for the measures to satisfy COM and UR

\begin{tabular}{|c|c|c|}
\hline Measure & $\mathrm{COM}$ & UR \\
\hline \multicolumn{3}{|l|}{ Panel A: Direct measures } \\
\hline (a) $y_{l}^{c 1}=\widehat{g}^{C}\left(\mathbf{c}_{\mathbf{l}}, 0\right)$ & $\mathrm{C} 1 \mathrm{M}$ & + \\
\hline (b) $y_{l}^{c 2}=\widehat{g}^{E}\left(\overline{\mathbf{e}}, \widehat{u}_{l}^{E}\right)$ & + & - \\
\hline (c) $y_{l}^{c 3}=\widehat{g}\left(\mathbf{c}_{\mathbf{l}}, \overline{\mathbf{e}}, 0\right)$ & $\begin{array}{c}\mathrm{E} \mu \text { and } \mathrm{C} 1 \mathrm{M} \\
\mathrm{COR} 0 \text { and } \mathrm{C} 1 \mathrm{M}\end{array}$ & $\mathrm{E} \mu$ and $\mathrm{COR} 0$ \\
\hline (d) $y_{l}^{c 4}=\widehat{g}\left(\mathbf{c}_{\mathbf{l}}, \overline{\mathbf{e}}, \widehat{u}_{l}\right)$ & COR0 & - \\
\hline (e) $y_{i}^{c 5}=\frac{1}{\left|N_{i}\right|} \sum_{k \in N_{i}} \widehat{g}\left(\mathbf{c}_{\mathbf{k}}, \mathbf{e}_{\mathbf{k}}, 0\right)$ & COR0 and C1M & COR0 \\
\hline Panel B: Indirect measures & & \\
\hline (f) $y_{l}^{E O 1}=\widehat{g}^{E}\left(\mathbf{e}_{\mathbf{l}}, 0\right)$ & + & - \\
\hline (g) $y_{l}^{E O 2}=\widehat{g}^{C}\left(\overline{\mathbf{c}}, \widehat{u}_{l}^{C}\right)$ & - & - \\
\hline (h) $y_{l}^{E O 3}=\widehat{g}\left(\overline{\mathbf{c}}, \mathbf{e}_{\mathbf{l}}, 0\right)$ & $\mathrm{C} \mu$ and COR0 & - \\
\hline (i) $y_{l}^{E O 4}=\widehat{g}\left(\overline{\mathbf{c}}, \mathbf{e}_{\mathbf{l}}, \widehat{u}_{l}\right)$ & - & - \\
\hline (j) $y_{i}^{E O 5}=\frac{1}{\left|N_{\cdot i}\right|} \sum_{k \in N_{. i}} \widehat{g}\left(\mathbf{c}_{\mathbf{k}}, \mathbf{e}_{\mathbf{k}}, 0\right)$ & COR0 & - \\
\hline
\end{tabular}

Note: A "+" means that the measure always satisfies the property in the column; a "-" means that the measure does not satisfy the property under any of the assumptions considered.

Table 2 contains three types of results. First, the Table shows that some measures always satisfy one of the properties. If one wants a measure to satisfy COM, one can use measure (b) or (f); if one wants a measure to satisfy UR, one can use (a). To see the latter, observe that to construct counterfactual (a) only circumstances are included in the regression. UR involves a Pigou-Dalton transfer between individuals having the same circumstances. From Lemma 1, this has no effect on the least squares estimator of the coefficient vector. Therefore, the counterfactual, and the inequality in the counterfactual will not be affected. The reason why measure (f), based on a regression including efforts only, satisfies COM is similar: the Pigou Dalton transfer between individuals with the same efforts does not affect the least squares estimator of the coefficient vector, such that the estimated counterfactual is unaffected. The transfer decreases inequality in the actual income distribution, such that the indirect measure of inequality of opportunity decreases. Counterfactual (b) is also based on a regression containing only efforts. From Lemma 1, a transfer between individuals having the same efforts has no effect on the estimated coefficient vector. However, the transfer affects the estimated random term for 2 individuals: the estimated random term for individual $i$ decreases with the amount $\delta$, for individual $j$ it increases with the same amount. Hence, inequality in the constructed counterfactual decreases and the measure satisfies COM.

Second, the Table provides sufficiency conditions under which some measures satisfy the principles. One-dimensionality of circumstances is a condition that occurs frequently in the Table as one of the sufficiency conditions for COM. Consider, for instance, the popular counterfactual (a), and a PigouDalton transfer between individuals having the same efforts (but different circumstances). The scalar covariance matrix equals the variance of the onedimensional circumstance, which is strictly positive, such that its inverse is also 
strictly positive. Hence, provided $i$ had better circumstances than $j$, Lemma 1 says that the least squares estimated coefficient decreases. As a result, the inequality in the estimated counterfactual decreases and the measure satisfies COM. Absence of correlation between efforts and circumstances often helps to establish properties of the inequality measures when the counterfactual relies on $\mathbf{b}^{C E}$. Consider, for example, counterfactual (d). Observe that the constructed counterfactual is independent of the estimated coefficient vector for circumstances, as, for every individual, the effect of a change in this vector on the counterfactual due to its effect through $\mathbf{c}_{1}$ is exactly off-set by its effect on the estimated random term $\widehat{u}_{l}$. The coefficient vector for efforts has an effect (as the effect through $\overline{\mathbf{e}}$ is not off-set by the effect on the estimated random term as the latter depends on $\mathbf{e}_{\mathbf{l}}$ ). However, remember the discussion following Lemma 1. In the absence of correlation between efforts and circumstances, the estimated coefficient vector of efforts is not affected by the differences in circumstances between $i$ and $j$, such that also this effect drops out. As a result, the effect of a Pigou-Dalton transfer between individuals having the same efforts on the counterfactual is equal to the effect on the actual income distribution: a Pigou-Dalton transfer between $i$ and $j$. Hence, inequality in the counterfactual decreases, and the measure satisfies COM. Observe that, in the absence of correlation between efforts and circumstances (e) has the same properties as (a) and (j) as (f). Comparing the averaging procedures with the reference value procedures (compare $(\mathrm{e})$ with $(\mathrm{c})$, and $(\mathrm{j})$ with $(\mathrm{h})$ ), the averaging procedures satisfy properties under weaker assumptions than the reference value procedures. The use of average values as reference values by itself does not help to guarantee that an inequality of opportunity measure has desirable properties, but in conjunction with other assumptions the properties of the measure can sometimes be established. Consider counterfactual (c). As the estimated coefficient vector of efforts is multiplied into $\overline{\mathbf{e}}-\mu_{\mathbf{E}}$, with reference efforts equal to average efforts, the counterfactual is independent of the estimated coefficient vector of efforts. A transfer between individuals having a different value for the one-dimensional circumstance (but the same efforts) will affect the estimated value of its regression coefficient in a way proportional to the inverse of the variance of that circumstance multiplied by the difference in circumstance between $j$ and $i$; if $i$ had better circumstances than $j$, the estimated regression coefficient decreases, and the inequality in the counterfactual decreases, such that COM is satisfied. We verified that one-dimensionality of efforts does not help direct measures to satisfy UR. On the contrary, it ensures that inequality in the counterfactual is affected.

Third, some measures do not satisfy certain properties under any of the assumptions made. We clearly see Proposition 1 reflected in the Table: none of the indirect measures satisfies UR. We also see other negative results for the measures in which the random variation is used in the construction of the counterfactuals. The direct measures with counterfactuals (b) and (d) do not satisfy UR, as this would require that the inequality in the counterfactual does not change. However, the Pigou-Dalton transfer always changes the estimated random terms for individuals $i$ or $j$, affecting the counterfactual and thereby 
the inequality in the counterfactual. The indirect measures with counterfactuals (g) and (i) do not satisfy COM. The reason is that even in situations where the estimated coefficients do not change, the change in the estimated random terms has an effect on the value of the inequality measure for the counterfactual distribution that may be larger or smaller than the change in the value of the inequality measure for the actual income distribution.

We conclude this section with some relevant observations for practitioners. If one only has information on circumstances, such that only counterfactuals based on $\mathbf{b}^{C}$ can be constructed, measures that satisfy UR are available, but no measure that satisfies COM is available. The opposite occurs in case one only has information on efforts. Finally, there are only three measures of inequality of opportunity in Table 2 that are not dominated by any other, in the sense that there does not exist another measure that requires weaker assumptions to satisfy the properties. These are measures (a), (b) and (f).

\subsection{Loglinear least squares}

The equations that have to be estimated to construct the counterfactuals are now, for $A \in\{C, E, C E\}$,

$$
\log (\mathbf{Y})-\iota \mu_{\log (Y)}=\mathbf{X}^{A} \alpha^{A}+\mathbf{V}^{A}
$$

where $\mu_{\log (Y)}=\frac{1}{n} \sum_{i=1}^{n} \log \left(y_{i}\right)$ and the corresponding least squares estimator of $\alpha^{A}$ is

$$
\mathbf{a}^{A}=\left(\mathbf{X}^{A \prime} \mathbf{X}^{A}\right)^{-1} \mathbf{X}^{A \prime}\left[\log (\mathbf{Y})-\iota \mu_{\log (Y)}\right] .
$$

Also here, performing a Pigou-Dalton transfer $\delta$ from observation $i$ to $j$ changes the least squares estimate of the coefficient vector. Lemma 2 gives the relationship between $\widetilde{\mathbf{a}}^{A}$, the least squares estimate after the Pigou-Dalton transfer, and $\mathbf{a}^{A}$.

Lemma 2 A Pigou-Dalton transfer $\delta$ from observation $i$ to $j$ changes the least squares estimator of the coefficient vector into $\widetilde{\mathbf{a}}^{A}$, given by

$$
\widetilde{\mathbf{a}}^{A}=\mathbf{a}^{A}+\frac{1}{n}\left(\mathbf{S}^{A}\right)^{-1}\left[\left(\mathbf{x}_{\mathbf{j}}{ }^{A} \Delta_{j}+\mathbf{x}_{\mathbf{i}}{ }^{A} \Delta_{i}\right],\right.
$$

with $\mathbf{S}^{A}=\frac{1}{n} \mathbf{X}^{A \prime} \mathbf{X}^{A}, \mathbf{x}_{\mathbf{j}}{ }^{A}=\mathbf{X}^{A \prime} \mathbf{E}_{\mathbf{j}}, \mathbf{x}_{\mathbf{i}}{ }^{A}=\mathbf{X}^{A \prime} \mathbf{E}_{\mathbf{i}}, \Delta_{j}=\log \left(y_{j}+\delta\right)-\log \left(y_{j}\right)$ and $\Delta_{i}=\log \left(y_{i}-\delta\right)-\log \left(y_{i}\right)$.

In Lemma 2 we see an element that we did not encounter in Lemma 1: $\Delta_{j}$ and $\Delta_{i}$, which depend on $Y_{j}$ and $Y_{i}$, respectively. We know that $\Delta_{j}>0>\Delta_{i}$ and that $\left|\Delta_{j}\right|>\left|\Delta_{i}\right|$. We see two ingredients we also encountered in Lemma 1. First, the covariance matrix $\mathbf{S}^{A}$ plays exactly the same role as in Lemma 1. If $\mathbf{X}^{A}$ contains only one variable, the scalar $\left(\mathbf{S}^{A}\right)^{-1}$ will be positive. If both circumstances and efforts are included in $\mathbf{X}^{A}$, and these variables are not correlated, the estimated coefficient vector of circumstances (resp. efforts) 
is not influenced by the efforts (resp. circumstances) of $i$ and $j$. Second, the values of $\mathbf{x}_{\mathbf{j}}{ }^{A}$ and $\mathbf{x}_{\mathbf{i}}{ }^{A}$ are also present. How they affect the estimated coefficient vector depends not only on the covariance matrix of the variables in $\mathbf{X}^{A}$ but also on the values of $\Delta_{j}$ and $\Delta_{i}$. Hence, contrary to Lemma 1 , a transfer between two individuals with the same values for $\mathbf{x}_{\mathbf{j}}{ }^{A}$ and $\mathbf{x}_{\mathbf{i}}{ }^{A}$ affects the estimated coefficient vector. It will be this feature which destroys the positive results found in Table 1.

The estimated coefficient vector (21) is used to determine the counterfactuals (6)-(10) and (12)-(16) (see Lemma A3 in Appendix A3). The counterfactual is influenced because the Pigou-Dalton transfer has an effect on the estimated coefficients, and increases the mean log of income (Lemma A4 in Appendix A3). We can verify whether the way the inequality of opportunity measures respond to Pigou-Dalton transfers is in accordance with the prescriptions in the properties COM and UR. The result is proven in Appendix A.3 and stated in Proposition 2.

Proposition 3 Using a loglinear specification and the least squares estimate of the coefficient vector to construct the counterfactual, none of the measures of inequality of opportunity satisfies COM nor UR.

The strong results in Table 1 (i.e. that UR is satisfied by (a) and COM by (b) and (f)) relied on the fact that the vector of coefficient estimates is unaffected by a Pigou-dalton transfer between individuals $j$ and $i$ when $\mathbf{x}_{\mathbf{j}}{ }^{A}=$ $\mathbf{x}_{\mathbf{i}}{ }^{A}$. Due to the presence of $\Delta_{j}$ and $\Delta_{i}$ in Lemma 2, these results do not hold any longer. The results for which the absence of correlation is important also relied on the same principle. It remains true that due to the absence of correlation one part of the coefficient vector is not affected by differences in $\mathbf{x}_{\mathbf{j}}{ }^{A} \Delta_{j}$ and $\mathbf{x}_{\mathbf{i}}{ }^{A} \Delta_{i}$. However, the other part of the coefficient vector no longer drops out because that part of $\mathbf{x}_{\mathbf{j}}{ }^{A} \Delta_{j}$ no longer equals the corresponding part of $\mathbf{x}_{\mathbf{i}}{ }^{A} \Delta_{i}$. Finally, the other results in Table 1 relied on $\mathrm{C} 1 \mathrm{M}$. With onedimensional circumstances, from Lemma 2, the scalar

$$
\widetilde{a}^{A} \geq a^{A} \Leftrightarrow x_{i}^{A} \leq-x_{j}^{A} \frac{\Delta_{j}}{\Delta_{i}} .
$$

We show the implications of this expression in Figure 1.

Pigou-Dalton transfers that take place between individuals above and to the left of the line through the origin with slope $-\frac{\Delta_{j}}{\Delta_{i}}>1$ decrease the estimated coefficient $a^{A}$, thereby decreasing the inequality in the estimated counterfactual. The opposite occurs for transfers between individuals below that line. Hence, even transfers between individuals with the same circumstances $\left(x_{i}^{A}=x_{j}^{A}\right)$ will affect the inequality in the estimated counterfactual. Inequality in the estimated counterfactual increases (decreases) if they both have circumstances better (worse) than average. ${ }^{7}$

\footnotetext{
7 Figure 1 can be adjusted to illustrate the result for linear least squares. In that case $\Delta_{j}=-\Delta_{i}=\delta$, the red line coincides with the 45 degree line, and a Pigou-Dalton transfer between individuals with the same circumstances has no effect on the estimated coefficient.
} 


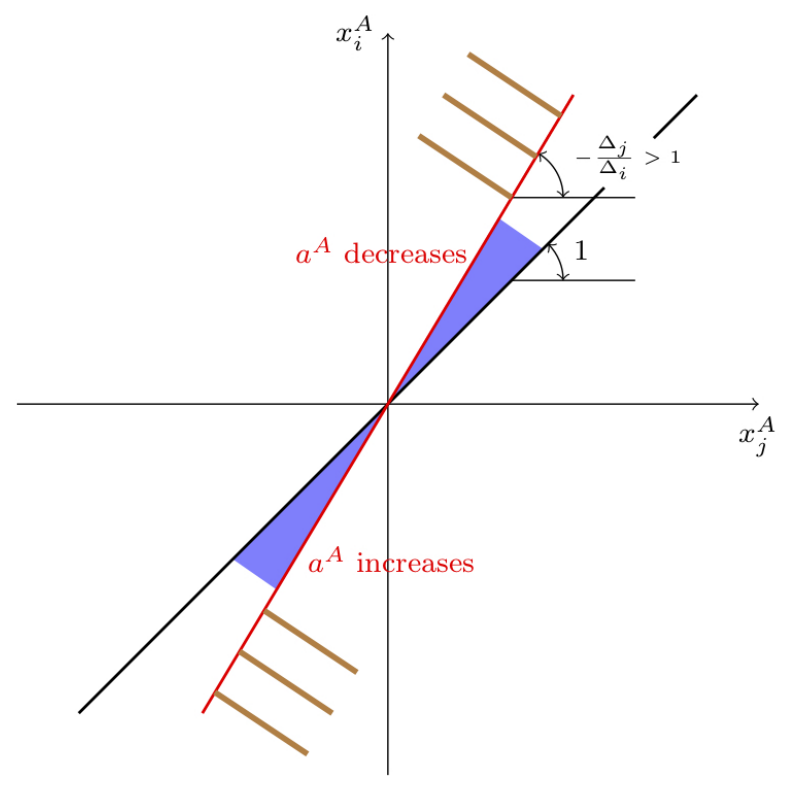

Fig. 1 The effect of a Pigou-Dalton transfer with loglinear least squares and onedimensional circumstances

We have seen that, when measuring inequality of opportunity for income, a loglinear specification is standard (see the references in the Introduction). Proposition 3 suggests that, with a loglinear specification, none of the resulting measures satisfies any of the basic principles that a measure of inequality of opportunity should have. This is bad news for what has become standard practice. However, if one were to claim that the relevant outcome is not income, but the log of income (for instance because it might be assumed that individual utilities can be measured that way) and a measure of inequality in the distribution of the log of (counterfactual) incomes is used in (1) or (2), then the transfers in the properties should apply to transfers in log income, which means that we are in the framework of Section 4.2, and the results from Proposition 2 apply. ${ }^{8}$

More precisely, consider the factor proportional transfer principle, in which, with $\delta>0$ the income of the rich individual is divided by a factor $1+\delta$, while the income of the poor individual is multiplied by $1+\delta$, without resulting in a post transfer income for the rich individual that is lower than the post

8 Actually, the empirical literature finds estimates close to one of the constant elasticity of marginal utility (see e.g. Layard et al., 2008, Gandelman and Hernández-Murillo, 2013), which could give empirical support to a loglinear specification as the best approximation to modeling individual utility as a function of income. 
transfer income of the poor individual. ${ }^{9}$ Replacing the standard Pigou-Dalton transfer in the COM and UR properties by such factor proportional transfers, it becomes clear that use of the loglinear specification (20) and a measure of inequality in the distribution of the log of (counterfactual) incomes in (1) or (2) means that the results from Table 2 apply to these factor proportional transfer versions of the COM and UR properties. Observe that it is crucial that the inequality measure is defined on the distribution of the log of incomes. This is, as far as we are aware of, never done in the inequality of opportunity literature. The mean log deviation is commonly used, but it is not suited, as it is the average value of the log of mean income divided by income. The standard deviation of the log of incomes, or any standard inequality measure defined on the distribution of the log of incomes are alternatives that are more coherent with the basic intuitions of inequality of opportunity measurement.

When measuring inequality of opportunity for income, a loglinear specification is almost universally used, and usually one uses the direct measure with counterfactual (6). The loglinear specification is motivated by its empirical fit; the use of a specification involving only circumstances is motivated by the absence of data on efforts. The results in this paper provide an additional and important motivation for this approach: if, in the properties, the transfers are defined as factor proportional transfers, then none of the other approaches in the literature satisfies the COM and UR principle under weaker assumptions. Moreover, it dominates the only other approach that only needs information on incomes and circumstances (the indirect measure with counterfactual (13)), which requires in addition to one-dimensionality of circumstances, that reference circumstances are set equal to their empirical averages in order to satisfy COM.

That the standard procedure is not dominated by any other, and that it always satisfies UR (with factor proportional transfers) are strong arguments in favor of the standard practice. Without information on efforts, it is the best one can do, but if circumstances are not one-dimensional it does not satisfy COM. Hence, it gives priority to the UR principle above the COM principle. Our results show that there exist measures that satisfy factor proportional transfer versions of the COM property, such as the direct measure with counterfactual (7) or the indirect measure with counterfactual (12), but they require information on efforts.

\subsection{Non-parametric approaches}

The properties of the non-parametric procedures to construct counterfactuals are easy to derive. It is clear that the counterfactual defined in (11) is unchanged if a Pigou-Dalton transfer occurs between two individuals having the same circumstances. Hence it immediately follows that when this counterfactual is used in the direct approach (1), the resulting measure of inequality of

\footnotetext{
9 This principle is different from the proportional transfer principles considered in Fleurbaey and Michel (2001). We discuss this in Appendix B.
} 
opportunity satisfies UR always and hence cannot satisfy COM. The counterfactual defined in (17) is unchanged if a Pigou-Dalton transfer occurs between two individuals having the same efforts. Hence, as the inequality in the actual income distribution has decreased, it immediately follows that when this counterfactual is used in the indirect approach (2), the resulting measure of inequality of opportunity satisfies COM always and hence cannot satisfy UR. We summarize the results in the following Proposition.

Proposition 4 The non-parametric counterfactual (11) in the direct approach always satisfies UR and never satisfies COM. The non-parametric counterfactual (17) in the indirect approach always satisfies COM and never satisfies UR.

At this point, it is worth pointing out that Ferreira and Peragine (2016) recently proposed an alternative non-parametric method that satisfies COM. They construct a counterfactual in which every individual's income is divided by the average income of those that have the same effort as he does. Inequality of opportunity is then measured by a relative inequality index defined over this counterfactual.

\section{Conclusion}

The theoretical literature on inequality of opportunity formulates some basic properties that measures of inequality of opportunity should have. The principle of compensation pleads for a reduction of inequality between individuals that have the same efforts. Utilitarian reward says that a transfer between individuals having the same circumstances should not affect inequality of opportunity.

The empirical literature tries to quantify the amount of inequality of opportunity. It has evolved in ways rather disconnected from these principles. This is especially so in the dominant part of the literature, which measures inequality of opportunity by means of counterfactuals, estimated through statistical procedures that are common to model the outcome of interest, such as income, education or health. As a result, the theoretical properties of the measures are obscured, and many theorists feel uneasy with the empirical work.

Bridging the gap between the theoretical and the empirical literature was one of the main goals of this paper. We have shown that some counterfactuals based on estimates from a linear least square specification yield measures that satisfy some of the desirable properties. This set of counterfactuals can be extended if additional assumptions, which have been previously used or discussed in the literature, are imposed. Contrary to this, no single measure of inequality of opportunity satisfies the desirable properties when counterfactuals are based on estimates from a loglinear specification. This is worrying, as this specification is commonly used when one is interested in inequality of opportunity for income. However, provided one assumes logarithmic utility, 
transfers are defined in terms of utility, and a measure of inequality of the distribution of $\log$ of incomes is used, the previous results are restored. This is, provided a right inequality measure is used, an additional motivation for what has become the standard approach in the measurement of inequality of opportunity for income, which measures the inequality in a counterfactual distribution, constructed on the basis of a loglinear least squares regression of incomes on circumstances only. In terms of incomes, this approach always satisfies the factor proportional transfer version of the utilitarian reward principle, and provided circumstances are one-dimensional and monotonic, it also satisfies the factor proportional transfer version of compensation. It is not dominated by any of the other approaches we considered. Finally, when nonparametric averaging procedures are used to construct counterfactuals in the direct approach, the resulting measure of inequality of opportunity always satisfies utilitarian reward. Doing the same for the counterfactual in the indirect approach results in a measure of inequality of opportunity that satisfies compensation.

The choice of measurement method (direct or indirect) and, with parametric methods, the choice of the functional form used to estimate and construct the counterfactual interact to determine the measure of inequality of opportunity's properties. So far these choices were based exclusively on their convenience, goodness of fit or what one is used to in a particular context. One should be aware these choices affect the properties of the measure of inequality of opportunity.

Our paper is a first analysis of the issues involved, and it has several shortcomings. First, the only reward principle we considered was utilitarian reward. Liberal reward is the most prominent reward principle in the axiomatic literature on fair allocations (see, e.g., Bossert, 1995, and Fleurbaey, 2005), and fair social orderings (see, e.g., Fleurbaey and Maniquet, 2005, 2008 and 2011). It states that government taxes and transfers should respect differences in incomes that are due to differences in responsibility. Hence, to incorporate the idea of liberal reward, one also needs information on net transfers. It is well known that also liberal reward is incompatible with compensation see Bossert (1995) and Fleurbaey (1995). The axiomatic literature proceeded to formulate weakened versions of compensation and liberal reward and formulated redistribution mechanisms that satisfy these weakened versions (see, e.g., Bossert and Fleurbaey, 1996). Devooght (2008) and Almås et al. (2011) propose to use the income resulting from these redistribution mechanism to define a norm income distribution and to measure inequality of opportunity by aggregating the deviations of individual's actual incomes from their norm incomes. The computation of the norm incomes relies on counterfactuals that are estimated using similar methods as the ones described here. Hence similar issues to the ones studied here also arise for that approach. Second, the only compensation principle we analyzed was the Ex-Post Compensation principle COM. The alternative is Ex-Ante Compensation, which requires that a transfer from an individual $i$ that belongs to a better-off type to an individual $j$ that belongs to a worse-off type, meaning that the average income of those that 
have the same circumstances as $i$ is higher than the average income of those that have the same circumstances as $j$, should decrease inequality of opportunity. This principle does not require that the individuals between which the transfers take place have the same efforts. Hence, typically, the effect on the counterfactuals constructed using the parametric methods can go either way, depending on, for instance, whether the effort of $i$ is larger than of $j$ or the other way around. Hence the principle will not be satisfied, except when least squares is used, counterfactual (a) is constructed and C1M is assumed. The non-parametric method for the direct approach always satisfies the Ex-Ante Compensation principle. Third, we only considered the most commonly used econometric models found in the literature. Researchers are using more and more advanced techniques to construct counterfactuals. Which, if any, of the basic properties the resulting measures of inequality of opportunity have is an important topic for future work.

\section{Appendices}

\section{A Proofs}

\section{A.1 Notation}

Define, for all individuals $l \in N$ the following vectors:

$$
\begin{aligned}
\mathbf{x}_{\mathbf{l}}{ }^{C} & =\left[c_{l, 1}-\mu_{C 1} \ldots c_{l, d^{C}}-\mu_{C d^{C}}\right]^{\prime}, \\
\mathbf{x}_{\mathbf{l}}{ }^{E} & =\left[e_{l, 1}-\mu_{E 1} \ldots e_{l, d^{E}}-\mu_{E d^{E}}\right]^{\prime}, \\
\mathbf{x}_{\mathbf{l}}{ }^{C \bar{C}} & =\left[c_{l, 1}-\bar{c}_{1} \ldots c_{l, d^{C}}-\bar{c}_{d^{C}}\right]^{\prime}, \\
\mathbf{x}_{\mathbf{l}}{ }^{E \bar{E}} & =\left[e_{l, 1}-\bar{e}_{1} \ldots e_{l, d^{E}}-\bar{e}_{d^{E}}\right]^{\prime} .
\end{aligned}
$$

Hence, $\mathbf{x}_{\mathbf{1}}^{C}\left(\mathbf{x}_{\mathbf{1}}^{C \bar{C}}\right)$ is the $d^{C}-$ dimensional vector of the deviation of circumstances of individual $l$ from their mean (reference) values, and $\mathbf{x}_{\mathbf{1}}^{E}\left(\mathbf{x}_{\mathbf{l}}^{E \bar{E}}\right)$ is the $d^{E}-$ dimensional vector of the deviation of his efforts from their mean (reference) values. Next, define the $\left(d^{C}+d^{E}\right)-$ dimensional vectors

$$
\begin{aligned}
\mathbf{x}_{\mathbf{l}}{ }^{C E} & =\left[\begin{array}{ll}
c_{l, 1}-\mu_{C 1} \ldots c_{l, d^{C}}-\mu_{C d^{C}} & e_{l, 1}-\mu_{E 1} \ldots e_{l, d^{E}}-\mu_{E d^{E}}
\end{array}\right]^{\prime}, \\
\mathbf{x}_{\mathbf{l}}{ }^{C E} & =\left[\begin{array}{ll}
c_{l, 1}-\mu_{C 1} \ldots c_{l, d^{C}}-\mu_{C d^{C}} & \bar{e}_{1}-\mu_{E 1} \ldots \bar{e}_{d^{E}}-\mu_{E d^{E}}
\end{array}\right]^{\prime}, \\
\mathbf{x}_{\mathbf{l}}{ }^{\bar{C}} & =\left[\begin{array}{ll}
\bar{c}_{1}-\mu_{C 1} \ldots \bar{c}_{d^{C}}-\mu_{C d^{C}} & e_{l, 1}-\mu_{E 1} \ldots e_{l, d^{E}}-\mu_{E d^{E}}
\end{array}\right]^{\prime}, \\
\mathbf{x}_{\mathbf{l}}{ }^{0 E} & =\left[\begin{array}{ll}
0 \ldots 0 & e_{l, 1}-\bar{e}_{1} \ldots e_{l, d^{E}}-\bar{e}_{d^{E}}
\end{array}\right]^{\prime}, \\
\mathbf{x}_{\mathbf{l}}{ }^{C 0} & =\left[\begin{array}{ll}
c_{l, 1}-\bar{c}_{1} \ldots c_{l, d^{C}}-\bar{c}_{d^{C}} & 0 \ldots 0
\end{array}\right]^{\prime},
\end{aligned}
$$

and the $n$-dimensional vectors $\mathbf{E}_{\mathbf{i}}=\left[\begin{array}{lllll}0 \ldots 0 & 1 & 0 \ldots 0\end{array}\right]^{\prime}$ and $\iota=\left[\begin{array}{ll}1 \ldots 1\end{array}\right]^{\prime}$, such that $\mathbf{E}_{\mathbf{i}}$ has zeros everywhere, except for its $i$ - element which equals one and all elements in $\iota$ are equal to one. 


\section{A.2 Proof of Proposition 2}

Proof of Lemma 1 Define $\widetilde{\mathbf{Y}}=\mathbf{Y}+\delta\left(\mathbf{E}_{\mathbf{j}}-\mathbf{E}_{\mathbf{i}}\right)$, which is the vector $\mathbf{Y}$ after a Pigou-Dalton transfer of an amount $\delta$ from observation $j$ to $i$. After the transfer we estimate the equation in deviational form

$$
\widetilde{\mathbf{Y}}^{D}=\widetilde{\mathbf{Y}}-\iota \mu_{Y}=\mathbf{X}^{A} \widetilde{\beta}^{A}+\widetilde{\mathbf{U}}^{A}
$$

For the least squares estimate $\widetilde{\mathbf{b}}^{A}=\left(\mathbf{X}^{A^{\prime}} \mathbf{X}^{A}\right)^{-1} \mathbf{X}^{A \prime} \widetilde{\mathbf{Y}}^{D}$ we obtain

$$
\left(\mathbf{X}^{A \prime} \mathbf{X}^{A}\right)^{-1} \mathbf{X}^{A \prime} \mathbf{Y}^{D}+\left(\mathbf{X}^{A \prime} \mathbf{X}^{A}\right)^{-1} \mathbf{X}^{A \prime}\left(\mathbf{E}_{\mathbf{j}}-\mathbf{E}_{\mathbf{i}}\right)
$$

from which the expression in the Lemma follows immediately.

Lemma A1 Under the assumption of linearity and using the least squares estimator, the counterfactuals (6)-(10) and (12)-(16) become

$$
\begin{aligned}
& y_{l}^{c 1}=\mu_{Y}+\mathbf{x}_{\mathbf{l}}{ }^{C \prime} \mathbf{b}^{C}, \\
& y_{l}^{c 2}=\mu_{Y}+y_{l}^{D}-\mathbf{x}_{\mathbf{l}}{ }^{E \bar{E}} \mathbf{b}^{E} \text {, } \\
& y_{l}^{c 3}=\mu_{Y}+\mathbf{x}_{\mathbf{l}}{ }^{C \bar{E}{ }^{\prime}} \mathbf{b}^{C E}, \\
& y_{l}^{c 4}=\mu_{Y}+y_{l}^{D}-\mathbf{x}_{\mathbf{l}}{ }^{0 E \prime} \mathbf{b}^{C E} \text {, } \\
& y_{l}^{c 5}=\mu_{Y}+\frac{1}{\left|N_{l \cdot}\right|} \sum_{k \in N_{l} .} \mathbf{x}_{\mathbf{k}}{ }^{C E{ }^{\prime}} \mathbf{b}^{C E} \\
& y_{l}^{E O 1}=\mu_{Y}+\mathbf{x}_{\mathbf{l}}^{E \prime} \mathbf{b}^{E}, \\
& y_{l}^{E O 2}=\mu_{Y}+y_{l}^{D}-\mathbf{x}_{\mathbf{l}}{ }^{C \bar{C} \prime} \mathbf{b}^{C} \text {, } \\
& y_{l}^{E O 3}=\mu_{Y}+\mathbf{x}_{\mathbf{l}}{ }^{\bar{C}{ }^{E \prime}} \mathbf{b}^{C E}, \\
& y_{l}^{E O 4}=\mu_{Y}+y_{l}^{D}-\mathbf{x}_{\mathbf{l}}^{C 0 \prime} \mathbf{b}^{C E} \text {. } \\
& y_{l}^{E 05}=\mu_{Y}+\frac{1}{\left|N_{\cdot l}\right|} \sum_{k \in N_{\cdot l}} \mathbf{x}_{\mathbf{k}}{ }^{C E \prime} \mathbf{b}^{C E}
\end{aligned}
$$

Proof of Lemma A1 Equations (A.2),(A.4), (A.6), (A.7), (A.9) and (A.11) are straightforward. The others are only slightly more complicated. We prove (A.3); the proof of the others is analogous. From (7), with the linear specification, and, for all individuals $l \in N$, $\mathbf{x}_{\mathbf{l}}{ }^{\bar{E}} \mu_{E}=\left[\bar{e}_{1}-\mu_{E 1} \ldots \bar{e}_{d^{E}}-\mu_{E d^{E}}\right]^{\prime}$, we have

$$
\begin{aligned}
y_{l}^{c 2} & =\mu_{Y}+\mathbf{x}^{\bar{E} \mu_{E}{ }^{\prime}} \mathbf{b}^{E}+\widehat{u}_{l}^{R} \\
& =\mu_{Y}+\mathbf{x}^{\bar{E} m u_{E} \mathbf{b}^{E}}+y_{l}^{D}-\mathbf{x}_{\mathbf{l}}{ }^{E \prime} \mathbf{b}^{E} \\
& =\mu_{Y}+y_{l}^{D}-\left(\mathbf{x}_{\mathbf{l}}^{E \prime}-\mathbf{x}^{\bar{E} \mu_{E}{ }^{\prime}}\right) \mathbf{b}^{E} \\
& =\mu_{Y}+y_{l}^{D}-\mathbf{x}_{\mathbf{l}}^{E \bar{E} \mathbf{b}^{E}}
\end{aligned}
$$

which is expression (A.3).

Using this Lemma, that the covariance matrix of the variables in $\mathbf{X}^{A}, \mathbf{S}^{A}=\frac{1}{n} \mathbf{X}^{A^{\prime}} \mathbf{X}^{A}$, and adding a tilde to denote the counterfactuals after the transfer, it is easy to obtain the following expressions for the effect of the Pigou-Dalton transfers on the counterfactuals.

Lemma A2 The change in the estimated counterfactuals of a Pigou-Dalton transfer $\delta$ from observation $i$ to $j$ is

$$
\widetilde{y}_{l}^{c 1}-y_{l}^{c 1}=\frac{\delta}{n} \mathbf{x}_{\mathbf{l}}{ }^{\prime \prime}\left(\mathbf{S}^{C}\right)^{-1}\left(\mathbf{x}_{\mathbf{j}}{ }^{C}-\mathbf{x}_{\mathbf{i}}{ }^{C}\right),
$$




$$
\begin{aligned}
& \widetilde{y}_{l}^{c 2}-y_{l}^{c 2}=\widetilde{y}_{l}^{D}-y_{l}^{D}-\frac{\delta}{n} \mathbf{x}_{\mathbf{l}}^{E \bar{E}}{ }^{\bar{\prime}}\left(\mathbf{S}^{E}\right)^{-1}\left(\mathbf{x}_{j}^{E}-\mathbf{x}_{\mathbf{i}}^{E}\right), \\
& \widetilde{y}_{l}^{c 3}-y_{l}^{c 3}=\frac{\delta}{n} \mathbf{x}_{\mathbf{l}}^{C E^{\prime}}\left(\mathbf{S}^{C E}\right)^{-1}\left(\mathbf{x}_{\mathbf{j}}{ }^{C E}-\mathbf{x}_{\mathbf{i}}{ }^{C E}\right), \\
& \widetilde{y}_{l}^{c 4}-y_{l}^{c 4}=\widetilde{y}_{l}^{D}-y_{l}^{D}-\frac{\delta}{n} \mathbf{x}_{\mathbf{l}}{ }^{0{ }^{\prime}}\left(\mathbf{S}^{C E}\right)^{-1}\left(\mathbf{x}_{\mathbf{j}}{ }^{C E}-\mathbf{x}_{\mathbf{i}}{ }^{C E}\right), \\
& \widetilde{y}_{l}^{c 5}-y_{l}^{c 5}=\frac{\delta}{n} \frac{1}{\left|N_{l \cdot}\right|} \sum_{k \in N_{l}} \mathbf{x}_{\mathbf{k}}{ }^{C E^{\prime}}\left(\mathbf{S}^{C E}\right)^{-1}\left(\mathbf{x}_{\mathbf{j}}{ }^{C E}-\mathbf{x}_{\mathbf{i}}{ }^{C E}\right), \\
& \widetilde{y}_{l}^{E O 1}-y_{l}^{E O 1}=\frac{\delta}{n} \mathbf{x}_{\mathbf{l}}^{E \prime}\left(\mathbf{S}^{E}\right)^{-1}\left(\mathbf{x}_{\mathbf{j}}{ }^{E}-\mathbf{x}_{\mathbf{i}}{ }^{E}\right), \\
& \widetilde{y}_{l}^{E O 2}-y_{l}^{E O 2}=\widetilde{y}_{l}^{D}-y_{l}^{D}-\frac{\delta}{n} \mathbf{x}_{\mathbf{l}}^{C \bar{C} \prime}\left(\mathbf{S}^{C}\right)^{-1}\left(\mathbf{x}_{\mathbf{j}}^{C}-\mathbf{x}_{\mathbf{i}}^{C}\right), \\
& \widetilde{y}_{l}^{E O 3}-y_{l}^{E O 3}=\frac{\delta}{n} \mathbf{x}_{\mathbf{l}}{ }^{\bar{C} E^{\prime}}\left(\mathbf{S}^{C E}\right)^{-1}\left(\mathbf{x}_{\mathbf{j}}{ }^{C E}-\mathbf{x}_{\mathbf{i}}{ }^{C E}\right), \\
& \widetilde{y}_{l}^{E O 4}-y_{l}^{E O 4}=\widetilde{y}_{l}^{D}-y_{l}^{D}-\frac{\delta}{n} \mathbf{x}_{\mathbf{l}}^{C 0 \prime}\left(\mathbf{S}^{C E}\right)^{-1}\left(\mathbf{x}_{\mathbf{j}}{ }^{C E}-\mathbf{x}_{\mathbf{i}}{ }^{C E}\right) \text {. } \\
& \widetilde{y}_{l}^{E O 5}-y_{l}^{E O 5}=\frac{\delta}{n} \frac{1}{\left|N_{\cdot l}\right|} \sum_{k \in N_{\cdot l}} \mathbf{x}_{\mathbf{k}}^{C E^{\prime}}\left(\mathbf{S}^{C E}\right)^{-1}\left(\mathbf{x}_{\mathbf{j}}{ }^{C E}-\mathbf{x}_{\mathbf{i}}{ }^{C E}\right),
\end{aligned}
$$

Proof of Proposition 2 Due to the similarity of the proofs, we first prove parts (a) and (f) followed by parts (b) and (g), (c) and (h), and, finally, parts (d) and (i).

Consider part (a) of the Proposition and Equation (A.12). Observe that, $\sum_{l=1}^{n} x_{l}^{C}=0$, such that, from (A.12), $\sum_{l=1}^{n} \widetilde{y}_{l}^{c 1}=\sum_{l=1}^{n} y_{l}^{c 1}$. The mean of the counterfactual has not changed, and there is no need to normalize the counterfactual distribution to study the effects of a Pigou-Dalton transfer.

Take $\mathbf{x}_{\mathbf{j}}{ }^{C}=\mathbf{x}_{\mathbf{i}}{ }^{C}$. From (A.12), $\widetilde{y}_{l}^{c 1}=y_{l}^{c 1}$, such that the counterfactual has not changed. Hence, UR is satisfied. Take $\mathbf{x}_{\mathbf{j}}{ }^{E}=\mathbf{x}_{\mathbf{i}}{ }^{E}$. Under C1M, $\left(\mathbf{S}^{C}\right)^{-1}>0$ and $c_{i 1}>c_{j 1}$. From (A.12) we immediately have that the value of the counterfactual decreases (increases) for those observations for which $x_{l}^{C}>(<) 0$, i.e. for which $c_{l 1}>(<) \mu_{C 1}$. Hence, $I\left(\mathbf{Y}^{c 1}\right)$ decreases and the measure satisfies COM.

Consider part (f) of the Proposition, and Equation (A.17). Observe that, $\sum_{l=1}^{n} x_{l}^{E}=0$, such that, from (A.17), $\sum_{l=1}^{n} \widetilde{y}_{l}^{E O 1}=\sum_{l=1}^{n} y_{l}^{E O 1}$. The mean of the counterfactual has not changed, and there is no need to normalize the counterfactual distribution to study the effects of a Pigou-Dalton transfer.

Take $\mathbf{x}_{\mathbf{j}}^{E}=\mathbf{x}_{\mathbf{i}}{ }^{E}$. From (A.17), $\widetilde{y}_{l}^{E O 1}=y_{l}^{E O 1}$, such that the counterfactual has not changed. However, the Pigou-Dalton transfer decreases the inequality in the income vector $\mathbf{Y}$, such that $I(\mathbf{Y})$ decreases, and thus $I(\mathbf{Y})-I\left(\mathbf{Y}^{E O 1}\right)$ decreases. Hence COM is satisfied. Take $\mathbf{x}_{\mathbf{j}}{ }^{C}=\mathbf{x}_{\mathbf{i}}{ }^{C}$. In case $d^{E}=1,\left(\mathbf{S}^{E}\right)^{-1}>0$. Let $e_{i 1}>e_{j 1}$. From (A.17), we have that for those observations for which $e_{l 1}>(<) \mu_{E 1}$, the counterfactual decreases (increases). Hence the transfer decreases inequality in $\mathbf{Y}^{E O 1}$. However, it also decreases the inequality in $\mathbf{Y}$ in a different manner, and the effect on $I(\mathbf{Y})-I\left(\mathbf{Y}^{E O 1}\right)$ is ambiguous, such that UR is not even satisfied with one-dimensional effort.

Consider part (b) of the Proposition and Equation (A.13). We have

$$
\frac{\sum_{l=1}^{n} \widetilde{y}_{l}^{c 2}}{n}=\frac{\sum_{l=1}^{n} y_{l}^{c 2}}{n}-\frac{\delta}{n}\left(\mu_{\mathbf{E}}-\overline{\mathbf{e}}\right)^{\prime}\left(\mathbf{S}^{E}\right)^{-1}\left(\mathbf{x}_{\mathbf{j}}{ }^{E}-\mathbf{x}_{\mathbf{i}}{ }^{E}\right) .
$$

Take $\mathbf{x}_{\mathbf{j}}{ }^{E}=\mathbf{x}_{\mathbf{i}}{ }^{E}$. From (A.22), the mean of the counterfactual has not changed. We then see from (A.13) that $\widetilde{y}_{l}^{c 2}-y_{l}^{c 2}=\widetilde{y}_{l}^{D}-y_{l}^{D}$, such that the inequality in the counterfactual declines and the measure satisfies COM. Take $\mathbf{x}_{\mathbf{j}}{ }^{C}=\mathbf{x}_{\mathbf{i}}{ }^{C}$. If $\overline{\mathbf{e}}=\mu_{\mathbf{E}}$, from (A.22), the mean of the counterfactual has not changed, and no normalization of the counterfactual is necessary to analyze the consequences of the Pigou-Dalton transfer. When $d^{E}=1,\left(\mathbf{S}^{E}\right)^{-1}>0$, and assuming that $e_{i 1}>e_{j 1}$, from (A.13) the change in the counterfactual is larger (smaller) than 
the change in the actual income distribution for those with $e_{l 1}>(<) \mu_{E 1}$, and inequality of opportunity changes. Hence the measure does not even satisfy UR under E $\mu$ with onedimensional efforts.

Consider part (g) of the Proposition and Equation (A.18). We have

$$
\frac{\sum_{l=1}^{n} \widetilde{y}_{l}^{E O 2}}{n}=\frac{\sum_{l=1}^{n} y_{l}^{E O 2}}{n}-\frac{\delta}{n}\left(\mu_{\mathbf{C}}-\overline{\mathbf{c}}\right)^{\prime}\left(\mathbf{S}^{C}\right)^{-1}\left(\mathbf{x}_{\mathbf{j}}{ }^{C}-\mathbf{x}_{\mathbf{i}}{ }^{C}\right) .
$$

Take $\mathbf{x}_{\mathbf{j}}{ }^{C}=\mathbf{x}_{\mathbf{i}}{ }^{C}$. From (A.23), the mean of the counterfactual has not changed. We then see, from (A.18) that $\widetilde{y}_{l}^{E O 2}-y_{l}^{E O 2}=\widetilde{y}_{l}^{D}-y_{l}^{D}$, such that the change in the counterfactual is identical to the change in the actual income distribution: in both distributions we get a Pigou-Dalton transfer from $i$ to $j$. However, nothing guarantees that the change in $I(\mathbf{Y})-$ $I\left(\mathbf{Y}^{E O 2}\right)$ will be the same, such that the measure does not satisfy UR. Take $\mathbf{x}_{\mathbf{j}}^{E}=\mathbf{x}_{\mathbf{i}}{ }^{E}$. If $\overline{\mathbf{c}}=\mu_{\mathbf{C}}$, from (A.23), the mean of the counterfactual has not changed, and no normalization of the counterfactual is necessary to analyze the consequences of the Pigou-Dalton transfer. With this reference value, with one-dimensional circumstances such that $\left(\mathbf{S}^{C}\right)^{-1}>0$, and assuming that $c_{i 1}>c_{j 1}$ (i.e. under $\mathrm{C} 1 \mathrm{M}$ ), from (A.18), we have that the change in the counterfactual is larger (smaller) than the change in the actual distribution for those with $c_{l 1}>(<) \mu_{C 1}$. However, this does not guarantee that $I(\mathbf{Y})-I\left(\mathbf{Y}^{E O 2}\right)$ decreases, and so even under these assumptions COM need not be satisfied. If $\overline{\mathbf{c}} \neq \mu_{\mathbf{C}}$, it follows from (A.23) that the mean of the counterfactual has changed. Under $\mathrm{C} 1 \mathrm{M}, c_{i 1}>c_{j 1}$, such that, if $\bar{c}_{1}<(>) \mu_{C 1}$, the mean increases (decreases), and this can counter the effect on inequality of opportunity that arises from the fact that, assuming that $c_{i 1}>c_{j 1}$, from (A.18), we have that the change in the counterfactual is larger (smaller) than the change in the actual distribution for those with $c_{l 1}>(<) \bar{c}_{1}$. A similar issue occurs in the following cases if the mean of the counterfactual changes; for that reason, we focus on cases where the mean remains constant.

The counterfactuals in (c), (d), (e), (h), (i) and (j) of the Proposition rely on estimates of $\mathbf{b}^{C E}$, such that $\mathbf{S}^{C E}$, the estimated covariance matrix of circumstances and efforts plays a role. Define the following matrix

$$
\left(\mathbf{S}^{C E}\right)^{-1}=\left[\begin{array}{ll}
\mathbf{A}^{C C} & \mathbf{A}^{C E} \\
\mathbf{A}^{E C} & \mathbf{A}^{E E}
\end{array}\right] .
$$

In case circumstances and efforts are not correlated, their covariance is zero, and $\mathbf{S}^{C E}$ is block diagonal. The inverse of a block-diagonal matrix is also block diagonal, such that, if efforts and circumstances are not correlated, $\mathbf{A}^{C E}=\left(\mathbf{A}^{E C}\right)^{\prime}$ contains only zeros.

Consider part (c) of the Proposition and Equation (A.14). Observe,

$$
\frac{\sum_{l=1}^{n} \widetilde{y}_{l}^{c 3}}{n}=\frac{\sum_{l=1}^{n} y_{l}^{c 3}}{n}+\frac{\delta}{n}\left(\overline{\mathbf{e}}-\mu_{\mathbf{E}}\right)^{\prime}\left[\mathbf{A}^{E C}\left(\mathbf{x}_{\mathbf{j}}^{C}-\mathbf{x}_{\mathbf{i}}^{C}\right)+\mathbf{A}^{E E}\left(\mathbf{x}_{\mathbf{j}}^{E}-\mathbf{x}_{\mathbf{i}}^{E}\right)\right] .
$$

Take $\overline{\mathbf{e}}=\mu_{\mathbf{E}}$. The mean of the counterfactual has not changed. First, with $\mathbf{x}_{\mathbf{j}}{ }^{C}=\mathbf{x}_{\mathbf{i}}{ }^{C}$, from (A.14),

$$
\widetilde{y}_{l}^{c 3}=y_{l}^{c 3}+\frac{\delta}{n} \mathbf{x}_{\mathbf{l}}^{C{ }^{C 1}} \mathbf{A}^{C E}\left[\mathbf{x}_{\mathbf{j}}^{E}-\mathbf{x}_{\mathbf{i}}^{E}\right] .
$$

If, in addition, $\mathbf{A}^{C E}=\mathbf{0}$, we get $\widetilde{y}_{l}^{c 3}=y_{l}^{c 3}$ : the transfer has no effect on the counterfactual $\mathbf{Y}^{c 3}$. Hence, in this case, the measure satisfies UR. Second, with $\mathbf{x}_{\mathbf{j}}{ }^{E}=\mathbf{x}_{\mathbf{i}}{ }^{E}$, from (A.14),

$$
\widetilde{y}_{l}^{c 3}=y_{l}^{c 3}+\frac{\delta}{n} \mathbf{x}_{\mathbf{l}}^{C \prime} \mathbf{A}^{C C}\left[\mathbf{x}_{\mathbf{j}}{ }^{C}-\mathbf{x}_{\mathbf{i}}{ }^{C}\right]
$$

If, in addition $d^{C}=1$ such that $A^{C C}>0$, and $c_{i 1}>c_{j 1}$, from (A.25), the counterfactual for those observations for which $c_{l 1}>(<) \mu_{C 1}$ decrease (increase), such that $I\left(\mathbf{Y}^{c 3}\right)$ decreases and the measure satisfies COM.

Take $\overline{\mathbf{e}} \neq \mu_{\mathbf{E}}, \mathbf{A}^{E C}=\mathbf{0}$ and $\mathbf{x}_{\mathbf{j}}{ }^{E}=\mathbf{x}_{\mathbf{i}}{ }^{E}$. From (A.24), the mean of the counterfactual has not changed, and, from (A.14), we obtain again (A.25), and the same conclusion follows: under $\mathrm{C} 1 \mathrm{M}$ the measure satisfies COM. 
Consider part (h) of the Proposition and Equation (A.19). Observe,

$$
\frac{\sum_{l=1}^{n} \widetilde{y}_{l}^{E O 3}}{n}=\frac{\sum_{l=1}^{n} y_{l}^{E O 3}}{n}+\frac{\delta}{n}\left(\overline{\mathbf{c}}-\mu_{\mathbf{C}}\right)^{\prime}\left[\mathbf{A}^{C C}\left(\mathbf{x}_{\mathbf{j}}{ }^{C}-\mathbf{x}_{\mathbf{i}}{ }^{C}\right)+\mathbf{A}^{C E}\left(\mathbf{x}_{\mathbf{j}}{ }^{E}-\mathbf{x}_{\mathbf{i}}{ }^{E}\right)\right] .
$$

Take $\overline{\mathbf{c}}=\mu_{\mathbf{C}}$. The mean of the counterfactual has not changed. First, with $\mathbf{x}_{\mathbf{j}}{ }^{E}=\mathbf{x}_{\mathbf{i}}{ }^{E}$, from (A.19),

$$
\widetilde{y}_{l}^{E O 3}=y_{l}^{E O 3}+\frac{\delta}{n} \mathbf{x}_{\mathbf{l}}^{E \prime} \mathbf{A}^{E C}\left[\mathbf{x}_{\mathbf{j}}^{C}-\mathbf{x}_{\mathbf{i}}^{C}\right] \text {. }
$$

If, in addition, $\mathbf{A}^{E C}=\mathbf{0}$, we get $\widetilde{y}_{l}^{E O 3}=y_{l}^{E O 3}$ : the transfer has no effect on the counterfactual. However, $I(\mathbf{Y})$ falls, hence $I(\mathbf{Y})-I\left(\mathbf{Y}^{E O 3}\right)$ decreases, and the measure satisfies COM. Second, with $\mathbf{x}_{\mathbf{j}}{ }^{C}=\mathbf{x}_{\mathbf{i}}{ }^{C}$, from (A.19),

$$
\widetilde{y}_{l}^{E O 3}=y_{l}^{E O 3}+\frac{\delta}{n} \mathbf{x}_{\mathbf{l}}^{E \prime} \mathbf{A}^{E E}\left[\mathbf{x}_{\mathbf{j}}^{E}-\mathbf{x}_{\mathbf{i}}^{E}\right]
$$

If, in addition $d^{E}=1$ such that $A^{E E}>0$, and $e_{i 1}>e_{j 1}$, from (A.27), we have that the counterfactual for those observations for which $e_{l 1}>(<) \mu_{E 1}$ decreases (increases). Hence the transfer decreases inequality in $\mathbf{Y}^{E O 3}$. However, since the inequality in $\mathbf{Y}$ decreases in a different way, the effect on $I(\mathbf{Y})-I\left(\mathbf{Y}^{E O 3}\right)$ cannot be determined and the measure does not even satisfy UR in the one-dimensional case.

Take $\overline{\mathbf{c}} \neq \mu_{\mathbf{C}}, \mathbf{A}^{C E}=0$ and $\mathbf{x}_{\mathbf{j}}{ }^{C}=\mathbf{x}_{\mathbf{i}}{ }^{C}$. From (A.26), the mean of the counterfactual has not changed, and, from (A.14), we obtain again (A.27), and the same conclusion follows: the measure does not satisfy UR even in the one-dimensional case.

Consider part (d) of the Proposition and Equation (A.15). Observe,

$$
\frac{\sum_{l=1}^{n} \widetilde{y}_{l}^{c 4}}{n}=\frac{\sum_{l=1}^{n} y_{l}^{c 4}}{n}-\frac{\delta}{n}\left(\mu_{\mathbf{E}}-\overline{\mathbf{e}}\right)^{\prime}\left[\mathbf{A}^{E C}\left(\mathbf{x}_{\mathbf{j}}{ }^{C}-\mathbf{x}_{\mathbf{i}}{ }^{C}\right)+\mathbf{A}^{E E}\left(\mathbf{x}_{\mathbf{j}}{ }^{E}-\mathbf{x}_{\mathbf{i}}{ }^{E}\right)\right] .
$$

Take $\overline{\mathbf{e}}=\mu_{\mathbf{E}}$. The mean of the counterfactual has not changed. First, with $\mathbf{x}_{\mathbf{j}}{ }^{C}=\mathbf{x}_{\mathbf{i}}{ }^{C}$, from (A.15),

$$
\widetilde{y}_{l}^{c 4}-y_{l}^{c 4}=\widetilde{y}_{l}^{D}-y_{l}^{D}-\frac{\delta}{n} \mathbf{x}_{\mathbf{l}}^{E \prime} \mathbf{A}^{E E}\left(\mathbf{x}_{\mathbf{j}}{ }^{E}-\mathbf{x}_{\mathbf{i}}{ }^{E}\right) .
$$

With this reference value, with one-dimensional efforts such that $A^{E E}>\mathbf{0}$, and assuming that $e_{i 1}>e_{j 1}$, from (A.29), the change in the counterfactual is larger (smaller) than the change in the actual income distribution for those with $e_{l 1}>(<) \mu_{E 1}$, and inequality of opportunity changes. Hence the measure does not satisfy UR. Second, with $\mathbf{x}_{\mathbf{j}}{ }^{E}=\mathbf{x}_{\mathbf{i}}{ }^{E}$, from (A.15)

$$
\widetilde{y}_{l}^{c 4}-y_{l}^{c 4}=\widetilde{y}_{l}^{D}-y_{l}^{D}-\frac{\delta}{n} \mathbf{x}_{\mathbf{l}}^{E \prime} \mathbf{A}^{E C}\left(\mathbf{x}_{\mathbf{j}}^{\mathbf{C}}-\mathbf{x}_{\mathbf{i}}^{\mathbf{C}}\right) .
$$

If, in addition, $\mathbf{A}^{E C}=\mathbf{0}, \widetilde{y}_{l}^{c 4}-y_{l}^{c 4}=\widetilde{y}_{l}^{D}-y_{l}^{D}$, such that inequality in the counterfactual decreases and the measure satisfies COM.

Take $\overline{\mathbf{e}} \neq \mu_{\mathbf{E}}, \mathbf{A}^{E C}=\mathbf{0}$ and $\mathbf{x}_{\mathbf{j}}{ }^{E}=\mathbf{x}_{\mathbf{i}}{ }^{E}$. From (A.28), the mean of the counterfactual has not changed, and, from (A.15), we obtain $\widetilde{y}_{l}^{c 4}-y_{l}^{c 4}=\widetilde{y}_{l}^{D}-y_{l}^{D}$, meaning that inequality decreased. Hence the measure satisfies COM.

Consider part (i) of the Proposition and Equation (A.20). Observe

$$
\frac{\sum_{l=1}^{n} \widetilde{y}_{l}^{E O 4}}{n}=\frac{\sum_{l=1}^{n} y_{l}^{E O 4}}{n}-\frac{\delta}{n}\left(\mu_{\mathbf{C}}-\overline{\mathbf{c}}\right)^{\prime}\left[\mathbf{A}^{C C}\left(\mathbf{x}_{\mathbf{j}}{ }^{C}-\mathbf{x}_{\mathbf{i}}{ }^{C}\right)+\mathbf{A}^{C E}\left(\mathbf{x}_{\mathbf{j}}{ }^{E}-\mathbf{x}_{\mathbf{i}}{ }^{E}\right)\right] .
$$

Take $\overline{\mathbf{c}}=\mu_{\mathbf{C}}$. The mean of the counterfactual has not changed. First, with $\mathbf{x}_{\mathbf{j}}{ }^{E}=\mathbf{x}_{\mathbf{i}}{ }^{E}$, from (A.20)

$$
\widetilde{y}_{l}^{E O 4}-y_{l}^{E O 4}=\widetilde{y}_{l}^{D}-y_{l}^{D}-\frac{\delta}{n} \mathbf{x}_{\mathbf{l}}^{C \prime} \mathbf{A}^{C C}\left(\mathbf{x}_{\mathbf{j}}{ }^{C}-\mathbf{x}_{\mathbf{i}}{ }^{C}\right) .
$$

If in addition $d^{C}=1$ such that $A^{C C}>0$, and $c_{i 1}>c_{j 1}$, by A.32, the change in the counterfactual for those observations for which $c_{l 1}>(<) \mu_{C 1}$ is larger (smaller) than the change in the actual income distribution. Hence inequality in the counterfactual increases 
more than the inequality in the actual income distribution. However, this does not guarantee that $I(\mathbf{Y})-I\left(\mathbf{Y}^{E O 4}\right)$ decreases, and so even under these assumptions COM need not be satisfied. Second, with $\mathbf{x}_{\mathbf{j}}{ }^{C}=\mathbf{x}_{\mathbf{i}}{ }^{C}$, from (A.20),

$$
\widetilde{y}_{l}^{E O 4}-y_{l}^{E O 4}=\widetilde{y}_{l}^{D}-y_{l}^{D}-\frac{\delta}{n} \mathbf{x}_{\mathbf{l}}^{C \prime} \mathbf{A}^{C E}\left(\mathbf{x}_{\mathbf{j}}{ }^{E}-\mathbf{x}_{\mathbf{i}}{ }^{E}\right) .
$$

If, in addition, $\mathbf{A}^{C E}=\mathbf{0}$, from (A.20), $\widetilde{y}_{l}^{E O 4}-y_{l}^{E O 4}=\widetilde{y}_{l}^{D}-y_{l}^{D}$, and the change in the counterfactual is identical to the change in the actual income distribution: in both distributions we get a Pigou-Dalton transfer from $i$ to $j$. However, nothing guarantees that the change in $I(\mathbf{Y})-I\left(\mathbf{Y}^{E O 4}\right)$ will be the same, such that the measure does not satisfy UR. Take $\overline{\mathbf{c}} \neq \mu_{\mathbf{C}}, \mathbf{A}^{C E}=\mathbf{0}$ and $\mathbf{x}_{\mathbf{j}}{ }^{C}=\mathbf{x}_{\mathbf{i}}{ }^{C}$. From (A.31), the mean of the counterfactual has not changed, and, from (A.15), we obtain again $\widetilde{y}_{l}^{E O 4}-y_{l}^{E O 4}=\widetilde{y}_{l}^{D}-y_{l}^{D}$. Again, the change in the counterfactual is identical to the change in the actual income distribution: in both distributions we get a Pigou-Dalton transfer from $i$ to $j$. However, nothing guarantees that the change in $I(\mathbf{Y})-I\left(\mathbf{Y}^{E O 4}\right)$ will be the same, such that the measure does not satisfy UR.

Consider part (e) of the Proposition and Equation (A.16). Observe,

$$
\frac{\sum_{l=1}^{n} \widetilde{y}_{l}^{c 5}}{n}=\frac{\sum_{l=1}^{n} y_{l}^{c 5}}{n}+\frac{\delta}{n} \frac{1}{n} \sum_{l=1}^{n} \frac{1}{\left|N_{l}\right|} \sum_{k \in N_{l} .} \mathbf{x}_{\mathbf{k}}^{C E^{\prime}}\left(\mathbf{S}^{C E}\right)^{-1}\left(\mathbf{x}_{\mathbf{j}}^{C E}-\mathbf{x}_{\mathbf{i}}{ }^{C E}\right) \text {. }
$$

Since $\sum_{l=1}^{n} \frac{1}{\mid N_{l . \mid}} \sum_{k \in N_{l}} \mathbf{x}_{\mathbf{k}}{ }^{C E \prime}=\mathbf{0}$, the $d^{C}+d^{E}$-dimensional null vector, the mean of the distribution has not changed. First, with $\mathbf{x}_{\mathbf{j}}{ }^{C}=\mathbf{x}_{\mathbf{i}}{ }^{C}$ and COR0 from (A.16),

$$
\widetilde{y}_{l}^{c 5}=y_{l}^{c 5}+\frac{\delta}{n} \frac{1}{\left|N_{l}\right|} \sum_{k \in N_{l} .} \mathbf{x}_{\mathbf{k}}^{E \prime} \mathbf{A}^{E E}\left(\mathbf{x}_{\mathbf{j}}^{E}-\mathbf{x}_{\mathbf{i}}^{E}\right) .
$$

The $i$-th element in the vector $\frac{1}{\left|N_{l}\right|} \sum_{k \in N_{l}} \mathbf{x}_{\mathbf{k}}{ }^{E \prime}$ is $\frac{1}{\left|N_{l .}\right|} \sum_{k \in N_{l} \text {. }} e_{k i}-\mu_{E i}$, the difference between the average value of effort $i$ of those that have the same circumstance as $l$ and the average value of effort $i$ in the population. Under COR0, the distribution of efforts conditional on circumstances is independent of the value of circumstances and equals the unconditional distribution. Hence $\frac{1}{N_{l} .} \sum_{k \in N_{l}} . \mathbf{x}_{\mathbf{k}}{ }^{E \prime}$ is equal to the $d^{E}$-dimensional null vector, $\widetilde{y}_{l}^{y c 5}=y_{l}^{y c 5}$ and the measure satisfies UR.

Second, with $\mathbf{x}_{\mathbf{j}}{ }^{E}=\mathbf{x}_{\mathbf{i}}{ }^{E}$, from (A.16),

$$
\widetilde{y}_{l}^{c 5}=y_{l}^{c 5}+\frac{\delta}{n} \frac{1}{\left|N_{l}\right|} \sum_{k \in N_{l} .} \mathbf{x}_{\mathbf{k}}^{C E \prime}\left[\begin{array}{l}
\mathbf{A}^{C C} \\
\mathbf{A}^{E C}
\end{array}\right]\left(\mathbf{x}_{\mathbf{j}}{ }^{C}-\mathbf{x}_{\mathbf{i}}{ }^{C}\right) .
$$

Under COR0, this reduces to

$$
\widetilde{y}_{l}^{c 5}=y_{l}^{c 5}+\frac{\delta}{n} \mathbf{x}_{\mathbf{l}}^{C \prime} A^{C C}\left(\mathbf{x}_{\mathbf{j}}{ }^{C}-\mathbf{x}_{\mathbf{i}}{ }^{C}\right) .
$$

With one-dimensional circumstances, therefore,

$$
\widetilde{y}_{l}^{c 5}=y_{l}^{c 5}+\frac{\delta}{n}\left[c_{l, 1}-\mu_{C 1}\right]\left(S^{C}\right)^{-1}\left(c_{j, 1}^{C}-c_{i, 1}^{C}\right),
$$

and the variance of the one-dimensional circumstance, $S^{C}>0$. C1M implies that $c_{j, 1}^{C}<c_{i, 1}^{C}$. Hence we get $\widetilde{y}_{l}^{c 5}<y_{l}^{c 5}$ for those with $c_{l, 1}>\mu_{C 1}$, which by $\mathrm{C} 1 \mathrm{M}$ is for those that have a higher than average income. Similarly, $\widetilde{y}_{l}^{c 5}>y_{l}^{c 5}$ for those with $c_{l, 1}<\mu_{C 1}$, which by C1M is for those that have a lower than average income. Hence we the transfer reduced inequality; COM is satisfied.

Consider part (i) of the Proposition and Equation (A.21). Observe,

$$
\frac{\sum_{l=1}^{n} \widetilde{y}_{l}^{E O 5}}{n}=\frac{\sum_{l=1}^{n} y_{l}^{E O 5}}{n}+\frac{\delta}{n} \frac{1}{n} \sum_{l=1}^{n} \frac{1}{\left|N_{\cdot l}\right|} \sum_{k \in N_{\cdot l}} \mathbf{x}_{\mathbf{k}}^{C E \prime}\left(\mathbf{S}^{C E}\right)^{-1}\left(\mathbf{x}_{\mathbf{j}}{ }^{C E}-\mathbf{x}_{\mathbf{i}}{ }^{C E}\right) \text {. }
$$


Since $\sum_{l=1}^{n} \frac{1}{\left|N_{. l}\right|} \sum_{k \in N_{. l}} \mathbf{x}_{\mathbf{k}}{ }^{C E \prime}=\mathbf{0}$, the $d^{C}+d^{E}$-dimensional null vector, the mean of the distribution has not changed. First, with $\mathbf{x}_{\mathbf{j}}{ }^{C}=\mathbf{x}_{\mathbf{i}}{ }^{C}$ and COR0, from (A.21),

$$
\widetilde{y}_{l}^{E O 5}=y_{l}^{E O 5}+\frac{\delta}{n} \mathbf{x}_{\mathbf{l}}^{E \prime} \mathbf{A}^{E E}\left(\mathbf{x}_{\mathbf{j}}^{E}-\mathbf{x}_{\mathbf{i}}^{E}\right) .
$$

Hence, under COR0 and with one-dimensional effort,

$$
\widetilde{y}_{l}^{E O 5}=y_{l}^{E O 5}+\frac{\delta}{n}\left[e_{l 1}-\mu_{E 1}\right]\left(\mathbf{S}^{E E}\right)^{-1}\left(\mathbf{x}_{\mathbf{j}}^{E}-\mathbf{x}_{\mathbf{i}}^{E}\right) .
$$

and it cannot be guaranteed that the change in inequality is equal to the change in inequality in the actual income distribution. Hence UR is not satisfied.

Second, with $\mathbf{x}_{\mathbf{j}}^{E}=\mathbf{x}_{\mathbf{i}}{ }^{E}$, from (A.21),

$$
\widetilde{y}_{l}^{E O 5}=y_{l}^{E O 5}+\frac{\delta}{n} \frac{1}{\left|N_{. l}\right|} \sum_{k \in N_{. l}} \mathbf{x}_{\mathbf{k}}^{C E \prime}\left[\begin{array}{l}
\mathbf{A}^{C C} \\
\mathbf{A}^{E C}
\end{array}\right]\left(\mathbf{x}_{\mathbf{j}}{ }^{C}-\mathbf{x}_{\mathbf{i}}{ }^{C}\right) .
$$

Under COR0, this reduces to

$$
\widetilde{y}_{l}^{E O 5}=y_{l}^{E O 5}+\frac{\delta}{n} \frac{1}{\left|N_{\cdot l}\right|} \sum_{k \in N_{\cdot l}} \mathbf{x}_{\mathbf{k}}^{C \prime} \mathbf{A}^{C C}\left(\mathbf{x}_{\mathbf{j}}{ }^{C}-\mathbf{x}_{\mathbf{i}}{ }^{C}\right) .
$$

The $i$-th element in the vector $\frac{1}{\left|N_{. l}\right|} \sum_{k \in N_{. l}} \mathbf{x}_{\mathbf{k}}{ }^{\prime \prime}$ is $\frac{1}{\left|N_{\cdot l}\right|} \sum_{k \in N_{\cdot l}} c_{k i}-\mu_{C i}$, the difference between the average value of circumstance $i$ of those that have the same effort as $l$ and the average value of circumstance $i$ in the population. Under COR0, the distribution of circumstances conditional on efforts is independent of the value of effort and equals the unconditional distribution. Hence $\frac{1}{\left|N_{. l}\right|} \sum_{k \in N_{. l}} \mathbf{x}_{\mathbf{k}}{ }^{\prime \prime}$ is equal to the $d^{C}$-dimensional null vector, $\widetilde{y}_{l}^{E O 5}=y_{l}^{E O 5}$, and the measure satisfies COM.

\section{A.3 Proof of Proposition 3}

Proof of Lemma 2 Define $\widetilde{\mathbf{Y}}=\mathbf{Y}+\delta\left(\mathbf{E}_{\mathbf{j}}-\mathbf{E}_{\mathbf{i}}\right)$, which is the vector $\mathbf{Y}$ after a Pigou-Dalton transfer of an amount $\delta$ from observation $j$ to $i$. After the transfer, for $A \in\{C, E, C E\}$, we estimate the equation in deviational form

$$
\log (\widetilde{\mathbf{Y}})-\iota \mu_{\log (\widetilde{Y})}=\mathbf{X}^{A} \widetilde{\alpha}^{A}+\widetilde{\mathbf{V}}^{A} .
$$

Observe that

$$
\log (\widetilde{\mathbf{Y}})=\log (\mathbf{Y})+\left(\log \left(y_{j}+\delta\right)-\log \left(y_{j}\right)\right) \mathbf{E}_{\mathbf{j}}+\left(\log \left(y_{i}-\delta\right)-\log \left(y_{i}\right)\right) \mathbf{E}_{\mathbf{i}},
$$

and that

$$
\mu_{\log (\widetilde{Y})}=\mu_{\log (Y)}+\frac{1}{n}\left[\log \left(y_{j}+\delta\right)-\log \left(y_{j}\right)+\log \left(y_{i}-\delta\right)-\log \left(y_{i}\right)\right],
$$

such that the least squares estimate,

$$
\widetilde{\mathbf{a}}^{A}=\left(\mathbf{X}^{A \prime} \mathbf{X}^{A}\right)^{-1} \mathbf{X}^{A \prime}\left[\log (\widetilde{\mathbf{Y}})-\iota \mu_{\log (\widetilde{Y})}\right],
$$

can be written as

$$
\begin{aligned}
\widetilde{\mathbf{a}}^{A}= & \mathbf{a}^{A}+\left(\mathbf{X}^{A \prime} \mathbf{X}^{A}\right)^{-1} \mathbf{X}^{A \prime} . \\
& {\left[\mathbf{E}_{\mathbf{j}}\left(\log \left(y_{j}+\delta\right)-\log \left(y_{j}\right)\right)+\mathbf{E}_{\mathbf{i}}\left(\log \left(y_{i}-\delta\right)-\log \left(y_{i}\right)\right)\right.} \\
& \left.(1 / n) \iota\left(\log \left(y_{j}+\delta\right)-\log \left(y_{j}\right)+\log \left(y_{i}-\delta\right)-\log \left(y_{i}\right)\right)\right] .
\end{aligned}
$$


As $\mathbf{S}^{A}=\frac{1}{n} \mathbf{X}^{A \prime} \mathbf{X}^{A}, \mathbf{X}^{A \prime} \mathbf{E}_{\mathbf{j}}=\mathbf{x}_{\mathbf{j}}{ }^{A}, \mathbf{X}^{A \prime} \mathbf{E}_{\mathbf{i}}=\mathbf{x}_{\mathbf{i}}{ }^{A}$ and $(1 / n) \mathbf{X}^{A \prime} \iota=0$, we obtain the expression in the Lemma.

Lemma A3 Under the assumption of loglinearity and using the least squares estimator, the counterfactuals (6)-(10) and (12)-(16) are defined by

$$
\begin{aligned}
& \log \left(y_{l}^{c 1}\right)=\mu_{\log (Y)}+\mathbf{x}_{\mathbf{l}}{ }^{\prime \prime} \mathbf{a}^{C}, \\
& \log \left(y_{l}^{c 2}\right)=\log \left(y_{l}\right)-\mathbf{x}_{\mathbf{l}}^{E \bar{E} \mathbf{a}^{E},} \\
& \log \left(y_{l}^{c 3}\right)=\mu_{\log (Y)}+\mathbf{x}_{\mathbf{l}}{ }^{C \bar{E} \mathbf{a}^{C E}}, \\
& \log \left(y_{l}^{c 4}\right)=\log \left(y_{l}\right)-\mathbf{x}_{\mathbf{l}}{ }^{0 E \prime} \mathbf{a}^{C E}, \\
& \log \left(y_{l}^{c 5}\right)=\mu_{\log (Y)}+\frac{1}{\left|N_{l}\right|} \sum_{k \in N_{l} .} \mathbf{x}_{\mathbf{k}}{ }^{C E \prime} \mathbf{a}^{C E}, \\
& \log \left(y_{l}^{E O 1}\right)=\mu_{\log (Y)}+\mathbf{x}_{\mathbf{l}}^{E \prime} \mathbf{a}^{E}, \\
& \log \left(y_{l}^{E O 2}\right)=\log \left(y_{l}\right)-\mathbf{x}_{\mathbf{l}}{ }^{C \bar{C} \mathbf{a}^{C}}, \\
& \log \left(y_{l}^{E O 3}\right)=\mu_{\log (Y)}+\mathbf{x}_{\mathbf{1}} \bar{C}{ }^{E \prime} \mathbf{a}^{C E}, \\
& \log \left(y_{l}^{E O 4}\right)=\log \left(y_{l}\right)-\mathbf{x}_{\mathbf{l}}{ }^{C 0 \prime} \mathbf{a}^{C E}, \\
& \log \left(y_{l}^{E O 5}\right)=\mu_{\log (Y)}+\frac{1}{\left|N_{\cdot l}\right|} \sum_{k \in N_{\cdot l}} \mathbf{x}_{\mathbf{k}}{ }^{C E \prime} \mathbf{a}^{C E} .
\end{aligned}
$$

Using Lemma 2 , it is straightforward to prove Lemma A4.

Lemma A4 Define $\Delta_{j}=\log \left(y_{j}+\delta\right)-\log \left(y_{j}\right)$ and $\Delta_{i}=\log \left(y_{i}-\delta\right)-\log \left(y_{i}\right)$. The change in the estimated counterfactuals of a Pigou-Dalton transfer $\delta$ from observation $i$ to $j$ is

$$
\begin{aligned}
& \log \left(\widetilde{y}_{l}^{c 1}\right)-\log \left(y_{l}^{c 1}\right)=\frac{1}{n}\left[\Delta_{j}+\Delta_{i}\right]+\frac{1}{n} \mathbf{x}_{\mathbf{l}}^{C \prime}\left(\mathbf{S}^{C}\right)^{-1}\left[\mathbf{x}_{\mathbf{j}}{ }^{C} \Delta_{j}+\mathbf{x}_{\mathbf{i}}{ }^{C} \Delta_{i}\right], \\
& \log \left(\widetilde{y}_{l}^{c 2}\right)-\log \left(y_{l}^{c 2}\right)=\log \left(\widetilde{y}_{l}\right)-\log \left(y_{l}\right)-\frac{1}{n} \mathbf{x}_{\mathbf{l}}^{E \bar{E} \prime}\left(\mathbf{S}^{E}\right)^{-1}\left[\mathbf{x}_{\mathbf{j}}{ }^{E} \Delta_{j}+\mathbf{x}_{\mathbf{i}}{ }^{E} \Delta_{i}\right], \\
& \log \left(\widetilde{y}_{l}^{c 3}\right)-\log \left(y_{l}^{c 3}\right)=\frac{1}{n}\left[\Delta_{j}+\Delta_{i}\right]+\frac{1}{n} \mathbf{x}_{\mathbf{l}}{ }^{C E}{ }^{\prime}\left(\mathbf{S}^{C E}\right)^{-1}\left[\mathbf{x}_{\mathbf{j}}{ }^{C E} \Delta_{j}+\mathbf{x}_{\mathbf{i}}{ }^{C E} \Delta_{i}\right], \\
& \log \left(\widetilde{y}_{l}^{c 4}\right)-\log \left(y_{l}^{c 4}\right)=\log \left(\widetilde{y}_{l}\right)-\log \left(y_{l}\right)-\frac{1}{n} \mathbf{x}_{\mathbf{l}}{ }^{0 E^{\prime}}\left(\mathbf{S}^{C E}\right)^{-1}\left[\mathbf{x}_{\mathbf{j}}{ }^{C E} \Delta_{j}+\mathbf{x}_{\mathbf{i}}{ }^{C E} \Delta_{i}\right], \\
& \log \left(\widetilde{y}_{l}^{c 5}\right)-\log \left(y_{l}^{c 5}\right)=\frac{1}{n}\left[\Delta_{j}+\Delta_{i}\right]+\frac{1}{n} \frac{1}{\left|N_{l \cdot}\right|} \sum_{k \in N_{l} .} \mathbf{x}_{\mathbf{k}}^{C E^{\prime}}\left(\mathbf{S}^{C E}\right)^{-1}\left[\mathbf{x}_{\mathbf{j}}{ }^{C E} \Delta_{j}+\mathbf{x}_{\mathbf{i}}{ }^{C E} \Delta_{i}\right], \\
& \log \left(\widetilde{y}_{l}^{E O 1}\right)-\log \left(y_{l}^{E O 1}\right)=\frac{1}{n}\left[\Delta_{j}+\Delta_{i}\right]+\frac{1}{n} \mathbf{x}^{E \prime}{ }^{E \prime}\left(\mathbf{S}^{E}\right)^{-1}\left[\mathbf{x}_{\mathbf{j}}{ }^{E} \Delta_{j}+\mathbf{x}_{\mathbf{i}}{ }^{E} \Delta_{i}\right], \\
& \log \left(\widetilde{y}_{l}^{E O 2}\right)-\log \left(y_{l}^{E O 2}\right)=\log \left(\widetilde{y}_{l}\right)-\log \left(y_{l}\right)-\frac{1}{n} \mathbf{x}_{\mathbf{l}}^{C C^{\prime}}\left(\mathbf{S}^{C}\right)^{-1}\left[\mathbf{x}_{\mathbf{j}}{ }^{C} \Delta_{j}+\mathbf{x}_{\mathbf{i}}{ }^{C} \Delta_{i}\right], \\
& \log \left(\widetilde{y}_{l}^{E O 3}\right)-\log \left(y_{l}^{E O 3}\right)=\frac{1}{n}\left[\Delta_{j}+\Delta_{i}\right]+\frac{1}{n} \mathbf{x}^{\bar{C}}{ }^{E \prime}\left(\mathbf{S}^{C E}\right)^{-1}\left[\mathbf{x}_{\mathbf{j}}^{C E} \Delta_{j}+\mathbf{x}_{\mathbf{i}}{ }^{C E} \Delta_{i}\right], \\
& \log \left(\widetilde{y}_{l}^{E O 4}\right)-\log \left(y_{l}^{E O 4}\right)=\log \left(\widetilde{y}_{l}\right)-\log \left(y_{l}\right)-\frac{1}{n} \mathbf{x}_{\mathbf{l}}{ }^{C 0 \prime}\left(\mathbf{S}^{C E}\right)^{-1}\left[\mathbf{x}_{\mathbf{j}}{ }^{C E} \Delta_{j}+\mathbf{x}_{\mathbf{i}}{ }^{C E} \Delta_{i}\right],
\end{aligned}
$$

Proof of Proposition 3 The left hand side in the equations of Lemma A4 give the percentage change in the estimated counterfactual. First, observe that, in (A.45), (A.47), (A.49), (A.50) (A.52) and (A.54), the first term is the same for all observations, and so has no effect on 
the inequality of the counterfactual, provided a relative measure of inequality is used. The problem to sign the effect of the transfer on the inequality measure is that the second term will be different for different observations, and will never disappear (not even when the transfer is between individuals having the same circumstances and/ or efforts - except, when, in addition they have the same income level, but in that case there is no PigouDalton transfer). Hence, these measures satisfy neither COM nor UR. Second, observe that in (A.46), (A.48) (A.51) and (A.53), the first term, $\log \left(\widetilde{y}_{l}\right)-\log \left(y_{l}\right)$, is zero for all observations, except for $i$ and $j$. Again, however, the problem is that the other term never vanishes, making it impossible to assess the effect of the transfer on the inequality in the counterfactual income distributions. Hence we obtain Proposition 3.

\section{B Alternative proportional transfer principles}

Let $y_{i}$ be individual $i$ 's income before the transfer, $y_{j}$ individual $j$ 's income before the transfer, $\widetilde{y}_{i}$ individual $i$ 's income after the transfer, and $\widetilde{y}_{j}$ individual $j$ 's income after the transfer. Throughout we require

$$
y_{i}>\widetilde{y}_{i} \geq \widetilde{y}_{j}>y_{j},
$$

such that the transfer goes from individual $i$ to individual $j$, and also after the transfer $i$ has at least as much income as $j$. Different proportional transfer principles impose different conditions on the transfers.

The Factor Proportional Transfer Principle (this paper) requires that, with $A>1$,

$$
\widetilde{y}_{i}=\frac{y_{i}}{A} \text { and } \widetilde{y}_{j}=y_{j} \cdot A \text {. }
$$

The Proportional Transfer Principle (Fleurbaey and Michel, 2001 p.4) requires that, with $\delta>0$,

$$
\widetilde{y}_{i}=y_{i}(1-\delta) \text { and } \widetilde{y}_{j}=y_{j}(1+\delta) .
$$

The Proportional Ex-Post Transfer Principle (Fleurbaey and Michel, 2001, p.4) requires that, with $\delta>0$,

$$
\widetilde{y}_{i}=\frac{y_{i}}{1+\delta} \text { and } \widetilde{y}_{j}=\frac{y_{j}}{1-\delta} .
$$

Assuming that the transfer described in the principle is desirable (because it decreases inequality), the following Proposition formulates the logical relationship between the three transfer principles.

Proposition C The Proportional Transfer Principle is stronger than the Factor Proportional Transfer Principle, which is stronger than the Proportional Ex-Post Transfer Principle.

Proof of Proposition $C$

(a) Comparison of (B.2) and (B.1). Consider the case where the transfer implies the same transfer in favor of the poor individual, i.e. $A=(1+\delta)$. In that case, the income after transfer for the rich person under (B.1) is higher than the income of the rich person under (B.2), as

$$
1-\delta^{2}=(1-\delta)(1+\delta)<1 \Longleftrightarrow \frac{y_{i}}{A}=\frac{y_{i}}{1+\delta}>y_{i}(1-\delta)
$$

Hence, all transfers that are acceptable under (B.2) are also acceptable under (B.1), but the reverse does not hold true. 
(b) Comparison of (B.1) and (B.3). Consider the case where the transfer implies the same transfer in favor of the poor individual, i.e. $A \prime=1 /(1-\delta)$. In that case, the income after transfer for the rich person under (B.3) is higher than the income of the rich person under (B.1), as

$$
1-\delta^{2}=(1-\delta)(1+\delta)<1 \Longleftrightarrow \frac{y_{i}}{1+\delta}>(1-\delta) y_{i}=\frac{y_{i}}{A \prime}
$$

Hence, all transfers that are acceptable under (B.1) are also acceptable under (B.3), but the reverse does not hold true.

Acknowledgements We thank the Editor, Marc Fleurbaey, anonymous referees of the Journal, Gerdie Everaert, José Luis Figueroa, François Maniquet, Erik Schokkaert and Alain Trannoy for many useful comments and suggestions.

\section{Conflict of interest}

The authors declare that they have no conflict of interest.

\section{References}

Almås I, Capellen AW, Lind J, Sorensen E, Tungodden B (2011) Measuring unfair (in)equality. J Public Econ 95:488-499

Arneson RJ (1989) Equality and equal opportunity for welfare. Phil Stud 56:77-93

Bossert W (1995) Redistribution mechanisms based on individual characteristics. Math Soc Sci 29:1-17

Bossert W, Fleurbaey M (1996) Redistribution and compensation. Soc Choice Welf 13:343356

Bourguignon F, Ferreira FHG, Menéndez M (2007) Inequality of opportunity in brazil. Rev Income Wealth 53:585-618

Brunori P, Peragine V, Serlenga L (2012) Fairness in education: the Italian university before and after the reform. Econ Educ Rev 31:764-777

Checchi D, Peragine V (2010) Inequality of opportunity in Italy. J Econ Inequal 8:429-450

Chetty R, Hendren N, Kline P, Saez E, Turner N (2014) Is the United States still a land of opportunity? recent trends in intergenerational mobility. Am Econ Rev Pap Proc 104:141147

Cohen JA (1989) On the currency of egalitarian justice. Ethics 99:906-944

Devooght K (2008) To each the same and to each his own: a proposal to measure responsibilty-sensitive income inequality. Economica 75:280-295

Dworkin R (1981a) What is equality? part 1: Equality of welfare. Phil Public Affairs 10:185245

Dworkin R (1981b) What is equality? part 2: Equality of resources. Phil Public Affairs 10:283-345

Ferreira FHG, Gignoux J (2011) The measurement of inequality of opportunity: Theory and an application to latin america. Rev Income Wealth 57:622-657, mimeo Development Research Group, The World Bank

Ferreira FHG, Gignoux J (2014) The measurement of educational inequality: achievement and opportunity. World Bank Econ Rev 28:210-246

Ferreira FHG, Peragine V (2016) Individual responsibility and equality of opportunity. In: Adler M, Fleurbaey M (eds) Oxford Handbook of Well-Being and Public Policy, Oxford University Press, Oxford, pp 746-784

Fleurbaey M (1995) The requisites of equal opportunity. In: Barnett WA, Moulin H, Salles M, Schofield NJ (eds) Social Choice, Welfare and Ethics, Cambridge University Press, Cambridge, pp 37-53 
Fleurbaey M (2008) Fairness, Responsibility and Welfare. Oxford University Press, Oxford

Fleurbaey M, Maniquet F (2005) Fair social orderings when agents have unequal production skills. Soc Choice Welf 24:93-127

Fleurbaey M, Maniquet F (2008) Fair social orderings. Econ Theor 34:24-45

Fleurbaey M, Maniquet F (2011) A theory of fairness and social welfare. Econometric Society Monograph, Cambridge University Press, Cambridge

Fleurbaey M, Michel P (2001) Transfer principles and inequality aversion, with an application to optimal growth. Math Soc Sci 42:1-11

Fleurbaey M, Peragine V (2013) Ex ante versus ex post equality of opportunity. Economica 80:118-130

Fleurbaey M, Schokkaert E (2009) Unfair inequalities in health and health care. J Health Econ 28:73-90

Foguel M, Veloso F (2014) Inequality of opportunity in daycare and preschool services in Brazil. J Econ Inequal 12:191-220

Gandelman H, Hernández-Murillo R (2013) What do happiness and health satisfaction data tell us about relative risk aversion? J Econ Psychol 39:301-312

García-Gómez P, Schokkaert E, Van Ourti T (2013) Reference value sensitivity of measures of unfair health inequality. Res Econ Inequality 21:1-36

Hassine NB (2012) Inequality of opportunity in Egypt. World Bank Econ Rev 26:265-295

Layard R, Mayraz G, Nickell S (2008) The marginal utility of income. J Public Econ 92:18461857

Luttens RI, Van de gaer D (2007) Lorenz dominance and non-welfaristic redistribution. Soc Choice Welf 28:281-302

Marrero GA, Rodríguez JG (2012) Inequality of opportunity in europe. Rev Income Wealth 58:597-621

Milanovic B (2015) Global inequality of opportunity: how much of our income is determined by where we live? Rev Econ Stat 97(2):452-460

Niehues J, Peichl A (2014) Upper bounds of inequality of opportunity: theory and evidence for Germany and the U.S. Soc Choice Welf 43:73-99

O'Neill D, Sweetman O, Van de gaer D (2000) Equality of opportunity and kernel density estimation: An application to intergenerational mobility. In: Fomby T, Hill R (eds) Advances in Econometrics, vol 14, Jai Press, Greenwich, pp 259-274

Pistolesi N (2009) Inequality of opportunity in the land of opportunities. J Econ Inequal 7:411-433

Ramos X, Van de gaer D (2016) Approaches to inequality of opportunity: principles, measures and evidence. J Econ Surveys 30:855-883

Rawls J (1971) A Theory of Justice. Oxford University Press: Oxford

Roemer JE (1993) A pragmatic theory of responsibility for the egalitarian planner. Phil Public Affairs 22:146-166

Roemer JE (1998) Equality of Opportunity. Harvard University Press, Cambridge

Roemer JE, Trannoy A (2015) Equality of opportunity. In: Atkinson AB, Bourgignon F (eds) Handbook of Income Distribution, vol 2A, North Holland, Amsterdam, pp 217-300

Rosa Dias P (2009) Inequality of opportunity in health: Evidence from a UK cohort study. Health Econ 18:1057-1074

Singh A (2012) Inequality of opportunity in earnings and consumption expenditure: the case of Indian men. Rev Income Wealth 58:79-106

Trannoy A, Tubeuf S, Jusot F, Devaux M (2010) Inequality of opportunities in health in france: A first pass. Health Econ 19:921-938

Van de gaer D (1993) Equality of Opportunity and Investment in Human Capital. KULeuven, Leuven

Van Parijs P (1995) Real freedom for all. Oxford University Press, Oxford 